ARTICLE

https://doi.org/10.1038/s41467-019-13986-6

\title{
Interaction variability shapes succession of synthetic microbial ecosystems
}

\author{
Feng Liu'1,2,3,4,12, Junwen Mao, 2,5,12, Wentao Kong ${ }^{1,2}$, Qiang Hua ${ }^{3,4}$, Youjun Feng ${ }^{6}$, Rashid Bashir ${ }^{1,7,8} \&$ \\ Ting $\mathrm{Lu}^{1,2,9,10,11 \star}$
}

Cellular interactions are a major driver for the assembly and functioning of microbial communities. Their strengths are shown to be highly variable in nature; however, it is unclear how such variations regulate community behaviors. Here we construct synthetic Lactococcus lactis consortia and mathematical models to elucidate the role of interaction variability in ecosystem succession and to further determine if casting variability into modeling empowers bottom-up predictions. For a consortium of bacteriocin-mediated cooperation and competition, we find increasing the variations of cooperation, from either altered labor partition or random sampling, drives the community into distinct structures. When the cooperation and competition are additionally modulated by $\mathrm{pH}$, ecosystem succession becomes jointly controlled by the variations of both interactions and yields more diversified dynamics. Mathematical models incorporating variability successfully capture all of these experimental observations. Our study demonstrates interaction variability as a key regulator of community dynamics, providing insights into bottom-up predictions of microbial ecosystems.

\footnotetext{
${ }^{1}$ Department of Bioengineering, University of Illinois at Urbana-Champaign, Urbana, IL, USA. ${ }^{2}$ Carl R. Woese Institute for Genomic Biology, University of Illinois at Urbana-Champaign, Urbana, IL, USA. ${ }^{3}$ State Key Laboratory of Bioreactor Engineering, East China University of Science and Technology, Shanghai, China. ${ }^{4}$ School of Bioengineering, East China University of Science and Technology, Shanghai, China. ${ }^{5}$ Department of Physics, Huzhou University, Huzhou, China. ${ }^{6}$ School of Medicine, Zhejiang University, Hangzhou, China. ${ }^{7}$ Micro and Nanotechnology Laboratory, University of Illinois at UrbanaChampaign, Urbana, IL, USA. ${ }^{8}$ Carle Illinois College of Medicine, Urbana, IL, USA. ${ }^{9}$ Department of Physics, University of Illinois at Urbana-Champaign, Urbana, IL, USA. ${ }^{10}$ Center for Biophysics and Quantitative Biology, University of Illinois at Urbana-Champaign, Urbana, IL, USA. ${ }^{11}$ National Center for Supercomputing Applications, University of Illinois at Urbana-Champaign, Urbana, IL, USA. ${ }^{12}$ These authors contributed equally: Feng Liu, Junwen Mao *email: luting@illinois.edu
} 
$\mathrm{M}$ icrobial communities are assemblages of multi-species microorganisms that live and interact with each other. They regulate the biogeochemical cycling of the planet ${ }^{1}$, fertilization of $\mathrm{crops}^{2}$, and metabolism of our human body ${ }^{3}$, thereby affecting profoundly the environment, agriculture and human health. As the dynamics of a community often underlies its emergent properties, being able to predict ecosystem succession is central to the elucidation of community organization and function $^{4}$, rational design of artificial ecosystems ${ }^{5-7}$ and introduction of intervention ${ }^{8}$. To that end, rapidly developed is bottom-up analysis ${ }^{9-12}$, which aims to determine community behaviors from the characterization of microbial interactionssuch as competition and cooperation-that are ubiquitous among microbes ${ }^{13}$. This approach is conceptually compelling, because it offers a systematic solution to capture emergent ecosystem properties and is potentially generalizable for both native and synthetic communities. In practice, although there are exciting successes in selected cases such as the utilization of the generalized Lotka-Volterra model for specific microbiomes ${ }^{14-16}$, predicting community dynamics from the bottom up remains as a grand challenge in general ${ }^{4}$.

One possible major cause of this challenge is pointed to the discrepancy between current modeling scheme and experimental observations. To date, bottom-up models commonly consider microbial interactions invariant and presume that determining interaction strength autonomously specifies community behaviors. Associated with these model developments are experimental efforts, which largely focus on the identification and measurement of interaction strength ${ }^{4,13}$. However, increasingly overwhelming evidences show that microbial interactions are highly variable, rather than static, in nature. Indeed, microbial interactions often change with environmental cues such as $\mathrm{pH}$, nutrient and stress ${ }^{17-27}$. For example, the mutualism between Escherichia coli and Rhodopseudomonas palustris is moderated by the toxicity of organic acids in the culture ${ }^{21}$; the antagonism from Pseudomonas aeruginosa to Staphylococcus aureus increases with ion depletion ${ }^{24}$. Microbial interactions are also subjective to the population of microorganisms generating the interactions as well as the presence of other species ${ }^{20,28-32}$. For instance, Lactococcus lactis produces nisin to suppress pathogens such as Staphylococcus aureus through quorum sensing of its own population ${ }^{31}$ while Enterococcus faecalis secrets cytolysin when sensing the presence of target cells ${ }^{32}$. Additionally, as cellular interactions are typically fulfilled through the production of metabolites and proteins-biochemical processes that are fundamentally stochastic $^{33-35}$, there are intrinsic fluctuations for all microbial interactions including those 'constant'. Recognizing this characteristic of microbial interactions, a handful of mathematical frameworks have been proposed to consider interaction variations ${ }^{36-40}$. In contrast, there is a lack of systematic experimental investigations that quantify the degree of variability for given microbial interactions. It also remains unclear to what extent such variations drive ecosystem succession and alter community structures and characteristics. Accordingly, it is unknown how the incorporation of variability into modeling shapes the predictive power of bottom-up mathematical modeling.

Here we hypothesize that variations of microbial interactions are a key modulator of community behaviors and characterizing and incorporating the variability empowers predictive understanding of ecosystem succession from the bottom up. To test the hypotheses, we design and build a set of synthetic three-strain microbial consortia, which involve both cooperation and competition, and use them as our experimental model systems. Compared to native ecologies, such synthetic communities possess a significantly reduced degree of complexity while offering the feasibility for mechanistic dissection and quantitative measurement ${ }^{7,41-49}$. In parallel, we develop mathematical models with an explicit incorporation of interaction variability to analyze ecosystem succession. For the consortium containing a variable cooperation, we quantify the variability of cooperation, elucidate the alteration of ecosystem dynamics arising from the variations, and demonstrate the power of variability-incorporated modeling in capturing community development. For the ecosystem whose cooperation and competition both fluctuate, more complex ecosystem dynamics arises but characterizing the variabilities again lead to successful succession predictions. Together, our results elucidate the role of interaction variability in regulating community dynamics, providing fundamental insights into bottomup understanding of microbial ecosystem succession.

\section{Results}

Creation of a cooperation between synthetic populations. We started by engineering a cooperation in synthetic populations because it is ubiquitous among microorganisms and critical to their organization ${ }^{50-52}$. Specifically, the interaction involves two engineered Lactococcus lactis strains, $\mathrm{C} \alpha$ and $\mathrm{C} \beta$, both of which harness the biosynthetic pathway of lcnG, a Class II two-subunit lactococcus bacteriocin (Fig. $1 \mathrm{a}$ and Methods) ${ }^{53,54}$. Here, Ca constitutively expresses $\operatorname{lag} A, \operatorname{lag} D$, and $\operatorname{lag} E$ which encode the peptide a precursor, $\mathrm{ABC}$ transporter and accessory protein of the pathway respectively, allowing the strain to synthesize and secret the $\alpha$ subunit of lcnG. Similarly, $C \beta$ constitutively expresses the pathway's $\beta$ precursor gene $\operatorname{lag} B$, transporter gene $\operatorname{lag} D$ and accessory protein gene $\operatorname{lag} E$, enabling the synthesis and secretion of the $\beta$ peptide, the other subunit of lcnG. In the extracellular milieu, the two subunits $\alpha$ and $\beta$ self-assemble into a bioactive antimicrobial which inhibits the growth of L. lactis strains. Through this fashion of division of labor, $C \alpha$ and $C \beta$ achieve a cooperation for successful lcnG production. As lcnG inhibits all $L$. lactis strains, the immunity gene $\operatorname{lag} C$ was introduced into both $\mathrm{C} \alpha$ and $\mathrm{C} \beta$ to confer them an immunity. Additionally, two reporter genes, yemGFP and mCherry, were loaded into Ca and $\mathrm{C} \beta$ respectively to enable the quantification of ecosystem dynamics.

To validate the cooperation, we conducted inhibition zone assays using the supernatants of $C \alpha$ and $C \beta$ monocultures (Methods). Four supernatant combinations, including blank culture (GM17 media supplemented with chloramphenicol) $(-/-)$, supernatant of $\mathrm{Ca}$ monoculture $(+/-)$, supernatant of $\mathrm{C} \beta$ monoculture $(-/+)$, and mix of the two supernatants $(+/+)$, were loaded into single wells in the solid agar plated with a lawn of lcnG-sensitive cells (L. lactis NZ9000 loaded with a chloramphenicol resistant gene $)^{55}$. Upon 8 hours (h) of incubation at $30^{\circ} \mathrm{C}$ (Methods), only the well loaded with the supernatant mix $(+/+)$ produced an inhibition zone (Fig. 1b). In addition, the four supernatant combinations were loaded into separate wells in agar plates covered with lcnG sensitive cells. We found that a clear inhibition zone formed between adjacent wells only when they were loaded with the $\mathrm{C} \alpha$ and $C \beta$ supernatants respectively (Fig. 1c). Together, the results confirmed two pieces of information. First, combination of $\mathrm{C} \alpha$ and $C \beta$ produced the active lcnG but individual strains alone did not. Second, peptides $\alpha$ and $\beta$ were able to autonomously assemble into an active bacteriocin without the need for any assistance.

Characterizing the variations of cooperation. Driven by diverse biotic and abiotic factors, microbial interactions are highly variable. Here we aimed to experimentally determine the variability of the cooperation originating from its division of labor nature. Specifically, to quantitate how labor partition, reflected by the cooperator ratio, affects the strength of cooperation, we grew the 
a

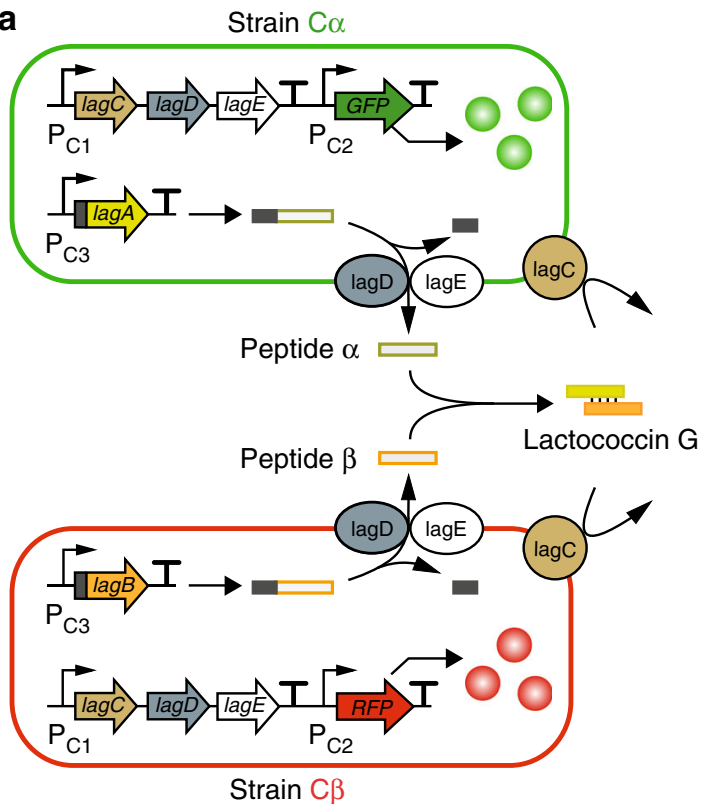

b

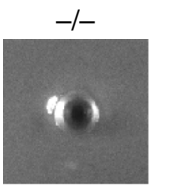

$+/-$

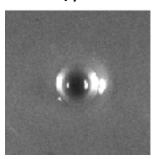

C

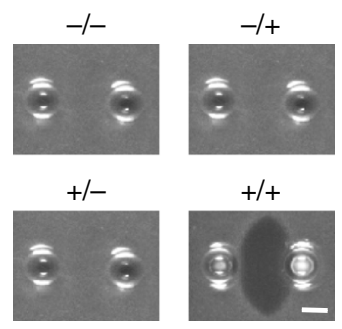

Fig. 1 An engineered cooperation between two bacterial strains. a Circuit design. The cooperative consortium consists of two $L$. lactis strains, $C \alpha$ and $C \beta$. $C \alpha$ carries three constitutively expressed genes $\operatorname{lag} A$, $\operatorname{lag} D$ and $\operatorname{lag} E$, allowing to synthesize and secret the $\alpha$ subunit of the bacteriocin lactococcin $\mathrm{G}$ (IcnG). $C \beta$ harbors three constitutively expressed genes lagB, lag $D$ and lagE, which enables the synthesis and secretion of the $\beta$ subunit of IcnG. The two subunits, $\alpha$ and $\beta$, autonomously assemble into IcnG that inhibits $L$. lactis strains. Meanwhile, $C \alpha$ and $C \beta$ both carry lagC, the IcnG immunity gene, and respectively have the fluorescence reporter genes yemGFP and mCherry. b Single-well inhibition zone assays. The four wells were loaded with blank culture (GM17 media supplemented with chloramphenicol) $(-/-)$, blank culture and $C \beta$ supernatant $(-/+), C \alpha$ supernatant and blank culture $(+/-)$, and $C \alpha$ and $C \beta$ supernatants $(+/+)$ accordingly. L. lactis NZ9000 loaded with a chloramphenicol resistant gene was used as an indicator strain. An inhibition zone was observed around the $(+/+)$ well containing the both supernatants. c Double-well inhibition zone assays. The left and right wells were loaded with blank culture and blank culture $(-/-)$, blank culture and $C \beta$ supernatant $(-/+), C \alpha$ supernatant and blank culture $(+/-)$, and $C \alpha$ and $C \beta$ supernatants $(+/$ + ) accordingly. L. lactis NZ9000 loaded with a chloramphenicol resistant gene was used as an indicator strain. An inhibition area was observed between the wells loaded with the $C \alpha$ and $C \beta$ supernatants respectively. In panels $\mathbf{b}$ and $\mathbf{c}$, scale bars, $3 \mathrm{~mm}$.

monocultures of $\mathrm{Ca}_{0}$ and $\mathrm{C} \beta_{0}$ (the reporter-free version of $\mathrm{C} \alpha$ and $\mathrm{C} \beta$ ), mixed their supernatants with varied ratios while fixing the total volume $(30 \mu \mathrm{L})$, and further used the mixes to perform inhibition zone experiments (Methods). Our results (Fig. 2a, top row) showed that, across the ratios from 30:1 to 1:30, the size of the inhibition zones varies from small to large and back to small with the largest occurring at the 1:1 ratio. As zone size correlates positively to a mix's lenG level and hence the strength of cooperation, the results suggested that initial labor partition can induce significant variations of the cooperation. Using the supernatants of $\mathrm{Ca}_{0}-\mathrm{C} \beta_{0}$ co-cultures with varied initial relative population abundance, we also observed the same dependence of the lcnG level on the initial population partition (Fig. 2a, bottom row). To quantitatively determine the variations, we further measured the relative lcnG level of each sample by normalizing its inhibition zones with that of $C \alpha \beta$, a lcnG-producing strain ( $L$. lactis MG1363 loaded with the complete lcnG pathway) (Fig. 2b, Methods, Supplementary Fig. 1).

In the above experiments, a low-high-low pattern of the cooperation strength (i.e., lcnG productivity) was observed with the maximum at the 1:1 labor partition. From a molecular perspective, we reasoned that the bell-shape variation of the cooperation is rooted in the 1:1 stoichiometric ratio of the $\alpha$ and $\beta$ subunits. We further speculated that the 1:1 initial ratio happened to be optimal because $C \alpha_{0}$ and $C \beta_{0}$ have a comparable growth rate and a comparable subunit productivity and, thus, the final ratio of the two peptides in a co-culture is solely determined by their initial densities. Supporting the speculation, the inhibition zone assays and $\operatorname{lcnG}$ quantifications for the $\mathrm{C} \alpha-\mathrm{C} \beta$ co-culture (Supplementary Fig. 2b, c) showed that the maximum was shifted towards a higher initial $C \beta$ abundance due to a slower growth rate of $\mathrm{C} \beta$ than $\mathrm{Ca}$ (Supplementary Fig. 3a). Such a growth reduction is a common consequence of heterologous protein production in microorganisms that is well documented in literature ${ }^{56,57}$. Meanwhile, the maximum for the monoculture mixes remained at 1:1 ratio (Supplementary Fig. 2a, c) because, in monocultures, $C \alpha$ and $C \beta$ had the same amount of nutrient and, thereby, produced a comparable level of subunits. To further confirm that the maximal cooperation is characterized by the stoichiometric ratio of the lcnG subunits, we derived new strains with altered peptide productivities, including $\mathrm{Ca}_{0 \mathrm{v} 2}$ with a doubled a productivity compared to $C a$, and $C \beta_{0 v 1 / 3}$ and $C \beta_{0 v 1 / 5}$ whose $\beta$ productivities are reduced to $1 / 3$ and $1 / 5$ of that of $C \beta_{0}$ (Supplementary Fig. 4). In theory, the combinations of $\mathrm{Ca}_{0}$ and $C \beta_{0 \mathrm{v} 1 / 3}, C \alpha_{0}$ and $C \beta_{0 \mathrm{v} 1 / 5}, C a_{0 \mathrm{v} 2}$ and $C \beta_{0 \mathrm{v} 1 / 3}$, and $C \alpha_{0 \mathrm{v} 2}$ and $C \beta_{0 \mathrm{v} 1 /}$ ${ }_{5}$ would shift the optimal ratios from 1:1 to 1:3, 1:5, 1:6, and 1:10 respectively, which were subsequently confirmed by the experiments (Fig. 2c).

For any microbial ecosystems, there are intrinsic random fluctuations of cellular populations arising from various stochastic processes $33-35,58,59$, which motivated us to quantify the variability of cooperation from intrinsic stochasticity by specifically examining the effects of sampling of initial populations (i.e., genetic drift ${ }^{60,61}$. We first inoculated the consortium from an initial culture (1:1 Ca-to- $C \beta$ ratio, 1.0 optical density at $600 \mathrm{~nm}$ (OD)) into fresh media through serial 1:10 dilution to generate samples with different initial ODs $\left(10^{-2}, 10^{-4}, 10^{-6}\right.$ and $\left.10^{-8}\right)$ (Fig. $2 \mathrm{~d}$ and Methods). Here, $10^{-8}$ was selected as the minimal initial OD since it is the minimal density for both $C \alpha$ and $C \beta$ to stably grow in monocultures (Supplementary Fig. 5). Next, we grew the cultures for the defined incubation time, collected their supernatants, and measured the lcnG levels (Methods). Figure 2e 
a

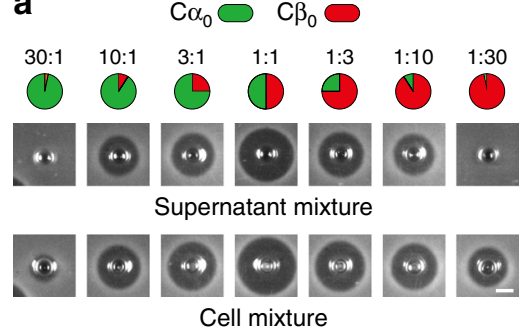

Cell mixture

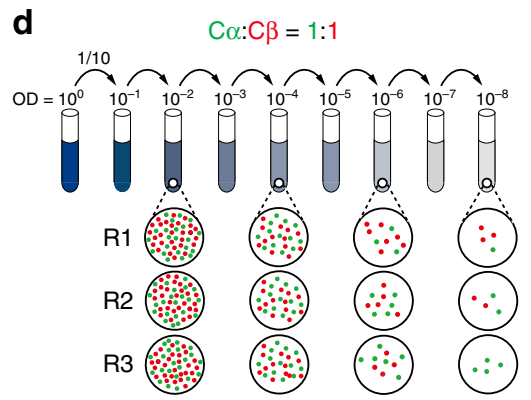

b
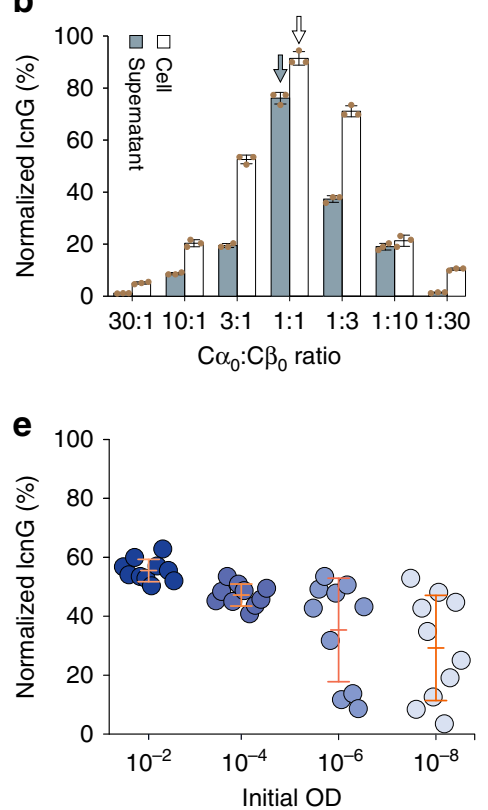

C

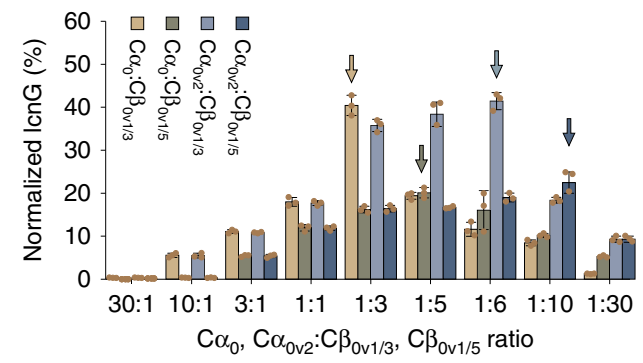

f

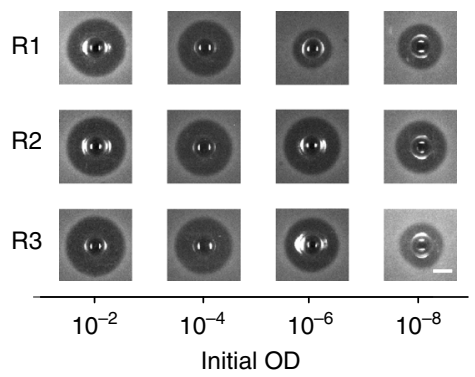

Fig. 2 Characterization of cooperation variations. a Representative inhibition zones formed by different ratios of supernatant mixes from the $C \alpha_{0}$ and $C \beta_{0}$ monocultures (top row) and the supernatants of the $C \alpha_{0}-C \beta_{0}$ co-cultures growing from different initial ratios (bottom row). $\mathbf{b}$ Relative lcnG levels in the monoculture supernatant mixes (white bars) and the co-culture supernatants (blue bars) $(n=3)$. Here, the IcnG concentration is normalized by the IcnG productivity of the single IcnG-producing strain $C \alpha \beta$. c Normalized IcnG levels in the supernatant mixes of monoculture $C \alpha_{0}$ and $C \beta_{0}$ variants $(n=3)$. C $\alpha_{0 v 2}$ is a $C \alpha$ variant that has a doubled peptide $\alpha$ productivity compared to $C \alpha_{0} . C \beta_{0 v 1 / 3}$ and $C \beta_{0 v 1 / 5}$ are two $C \beta_{0}$ variants, whose peptide $\beta$ productivities are $1 / 3$ and $1 / 5$ of that of $C \beta_{0}$. d Schematic illustration of population fluctuations during serial dilution. Variations of the cooperator ratio increase with reducing initial cell density, resulting in different abundances across replicates (R1, R2, and R3). e Normalized IcnG concentration of the C $\alpha$-C $\beta$ co-culture from different initial ODs but a fixed 1:1 ratio. Each circle represents the IcnG level from a single experiment. For each OD, there are a total of 10 replicates $(n=$ 10). $\mathbf{f}$ Representative inhibition zones formed by the consortium growing from different initial ODs. In panel $\mathbf{b}$, $\mathbf{c}$, and $\mathbf{e}$, bars and error bars correspond to means and s.d. In panels a and $\mathbf{f}$, scale bars, $3 \mathrm{~mm}$. Source Data available in the source data file.

shows the lcnG levels of the co-cultures normalized by the productivity of $\mathrm{C} \alpha \beta$, a single-strain $\mathrm{ln} G$ producer we created. We found that, with the reduction of initial OD, the mean lcnG productivity of the consortium decreased monotonically but, in the meanwhile, the sample-to-sample variation increased. Such a trend was also clearly observed in the inhibition zones formed by the co-cultures (Fig. $2 \mathrm{f}$ and Supplementary Fig. 6). By conducting the same experiments for the reporter-free version of the consortium, the $\mathrm{Ca}_{0}-\mathrm{C} \beta_{0}$ ecosystem (Supplementary Fig. 7), we confirmed that random sampling continued to serve as a key inducing factor of the variation.

Notably, partition alteration and random sampling are two independent sources of cooperation variations; however, they are intrinsically connected. The both alter cooperation strength by varying the division of labor among the cooperators, but the former is a controlled, deterministic alteration of the partition while the latter is unintended, stochastic alteration.

Ecosystem successions driven by cooperation variations. To elucidate the consequences of interaction variations on community behaviors, we designed a three-strain consortium composed of the two cooperators ( $\mathrm{Ca}$ and $\mathrm{C} \beta$ ) and a competitive third strain (Ks). Here, Ks is an engineered strain capable of constitutively secreting lactococcin A $(\mathrm{lnn} A)^{62}$, a bacteriocin that effectively kills all L. lactis strains including $\mathrm{C} \alpha$ and $C \beta$ unless immunized. Experimentally, Ks was built by introducing the lcnA pathway into the L. lactis MG1363 (Fig. 3a). To efficiently count Ks in the three-strain ecosystem, it was inserted with constitutively expressed gusA3, a beta-glucuronidase gene that enables colorimetric quantification upon the supplementation of X-Gluc ${ }^{63}$. As
Ks does not contain the $\operatorname{lcn} G$ immunity gene $\operatorname{lag} C$, it is sensitive to $\ln G$ cooperatively produced by $\mathrm{Ca}$ and $\mathrm{C} \beta$. Therefore, the community involves a cooperation between $\mathrm{Ca}$ and $\mathrm{C} \beta$ and a competition of Ks with $\mathrm{C} \alpha$ and $\mathrm{C} \beta$ (Fig. 3b).

Meanwhile, we hypothesized that predicting ecosystem behaviors requires mathematical models that take in account the variability of interaction. To test the hypothesis, we devised a dynamic ecosystem model using a systematic, bottom-up fashion (Methods, Supplementary Information 1). Briefly, we first constructed models of $\mathrm{C} \alpha$ and $\mathrm{C} \beta$ monocultures (Supplementary Equations 1-2, Supplementary Fig. 3b), and used them as modules to derive a model of $\mathrm{Ca}-\mathrm{C} \beta$ co-culture incorporating variability (Supplementary Equation 4) with their parameters specified with experiments (Supplementary Fig. 8). We then constructed a model of lcnA-producing Ks monoculture (Supplementary Equation 5) and determined its parameters experimentally (Supplementary Fig. 9a, b). Finally, by merging the models of the $\mathrm{C} \alpha-\mathrm{C} \beta$ co-culture model with the Ks monoculture and characterizing the inhibitions between the modules (Supplementary Equations 6-7, Supplementary Fig. 9c$\mathrm{f}$, we obtained a model for the $\mathrm{Ca}-\mathrm{C} \beta-\mathrm{Ks}$ consortium (Supplementary Equation 9). For batch fermentations starting from a fixed $(\mathrm{C} \alpha+\mathrm{C} \beta):$ Ks ratio $(2: 1)$, the model predicted that the ecosystem evolves temporally into Ks dominance at imbalanced cooperator partitions and Ks subordinate at close partitions (Fig. 3c), suggesting that the variations of cooperation can modulate the succession of the consortium dramatically.

To test the predictions, we experimentally assembled the consortium by mixing $\mathrm{C} \alpha, \mathrm{C} \beta$ and Ks with altered initial ratios but a fixed total OD $\left(10^{-2}\right)$ as our model construction (Methods). Consistent with the predictions, our fermentations showed that 
a

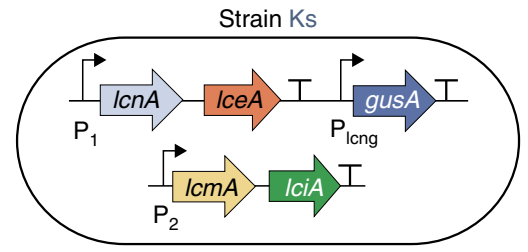

b

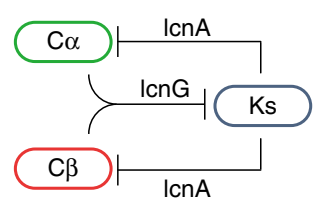

C
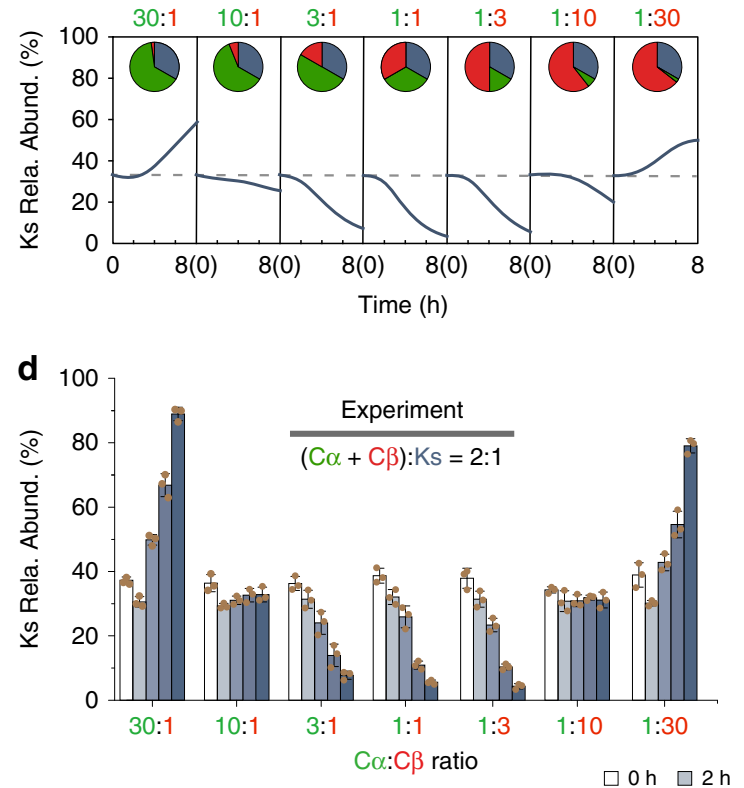

e

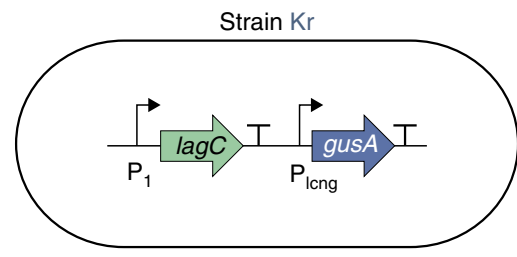

f

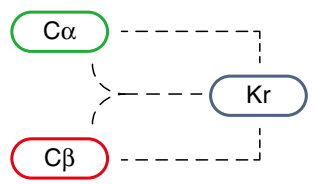

9

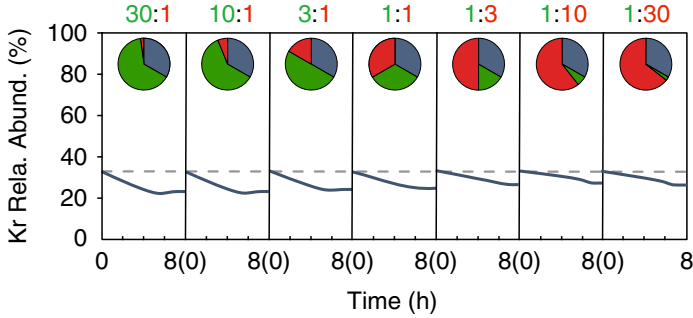

h
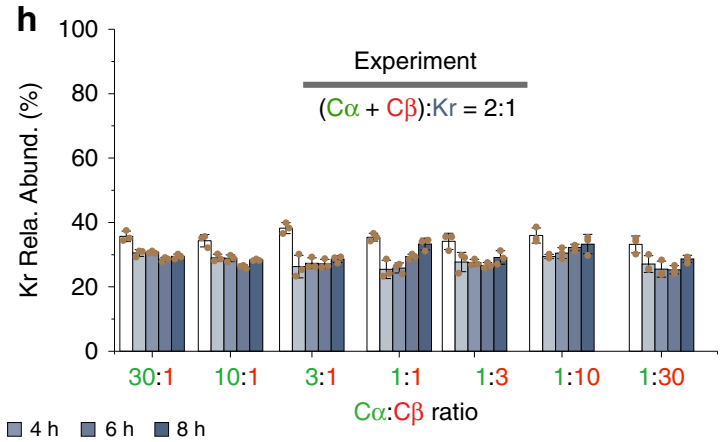

Fig. 3 Succession of a three-strain ecosystem driven by cooperation variations from labor partition. a Design of a competitive third strain Ks. The IcnA biosynthesis pathway, including the genes $I \mathrm{cn} A$, IceA, IciA, and IcmA, is placed under constitutive promoters for constant IcnA secretion. The gene gus $\mathrm{c} 3$ is also introduced for colorimetric quantification. $\mathbf{b} A$ three-strain consortium composed of $C \alpha, C \beta$, and Ks. $C \alpha$ and $C \beta$ cooperate to produce lcnG that inhibits $\mathrm{Ks}$; in turn, Ks secrets IcnA to oppose $\mathrm{C} \alpha$ and $\mathrm{C} \beta$. c , d Model-predicted (c) and experimentally measured (d) temporal dynamics of the Ks abundance in the $\mathrm{C} \alpha-C \beta-K s$ ecosystem. The initial $C \alpha: C \beta$ partition was varied across 30:1 to 1:30, but the total $(C \alpha+C \beta)$ : Ks ratio was fixed as 2:1. e Design of a control strain Kr. L. lactis MG1363 is loaded with the constitutively expressed lagC, the IcnG immunity gene, to confer resistance to IcnG and gus $A 3$ for colorimetric quantification. $\mathbf{f} A$ three-strain ecosystem composed of $\mathrm{C} \alpha, C \beta$ and $\mathrm{Kr}$. As $\mathrm{Kr}$ is deficient in IcnA production but resistant to IcnG, the consortium does not have active bacteriocin-mediated interactions. $\mathbf{g}$, $\mathbf{h}$ Model predictions ( $\mathbf{g}$ ) and experimental measures (h) of temporal dynamics of the $\mathrm{Kr}$ strain in the $\mathrm{C} \alpha$ $\mathrm{C} \beta-\mathrm{Kr}$ ecosystem. The initial $\mathrm{C} \alpha: C \beta$ partition was varied across 30:1 to 1:30, but the total $(C \alpha+C \beta)$ : $\mathrm{Kr}$ ratio was fixed as 2:1. Source Data available in the source data file.

the Ks percentage indeed increased over time and eventually became dominant when the $\mathrm{Ca}: \mathrm{C} \beta$ ratio was extremely imbalanced (30:1 and 1:30); in contrast, when their abundances were close $(3: 1,1: 1$, and $1: 3)$, the Ks percentage declined monotonically and diminished eventually (Fig. 3d). Opposite with the Ks percentage, the relative abundances of $\mathrm{C} \alpha$ and $\mathrm{C} \beta$ both decreased at imbalanced $C \alpha: C \beta$ ratios $(30: 1$ and 1:30) but increased when the ratio is close $(3: 1,1: 1$, and 1:3) (Supplementary Fig. 10a, b). Meanwhile, although the total OD of the ecosystem remained largely consistent regardless of the $\mathrm{Ca}: \mathrm{C} \beta$ ratios (Supplementary Fig. 8, circles), the lcnG level changed significantly (Supplementary Fig. 8, bars): it remained limited throughout the fermentations in imbalanced scenarios but accumulated rapidly at the balanced cases, consistent with our previous characterization (Fig. 2a-c). The correspondence between higher lcnG production (Supplementary Fig. 8) and lower Ks abundance (Fig. 3c, d) suggested a strong correlation between cooperation variations and diversified ecosystem succession.

To further confirm that it is a causal relationship between interaction variations and diversified succession, we designed a Ks variant, named $\mathrm{Kr}$, which is resistant to $\mathrm{ln} \mathrm{C}$ co-produced by $\mathrm{Ca}$ and $\mathrm{C} \beta$ and deficient in producing lcnA. Experimentally, $\mathrm{Kr}$ was established by removing the lcnA biosynthetic pathway from Ks while introducing the $\mathrm{ln} G$ immunity gene lagC (Fig. 3e, Supplementary Fig. 11). The mixture of $\mathrm{Ca}, \mathrm{C} \beta$ and $\mathrm{Kr}$ formed a control consortium where the cooperation and competition are both abolished (Fig. 3f). Our mathematical model (Methods, Supplementary Equation 11) predicted that, upon the same alterations of the initial $\mathrm{Ca}: \mathrm{C} \beta$ ratio, the structure of the 

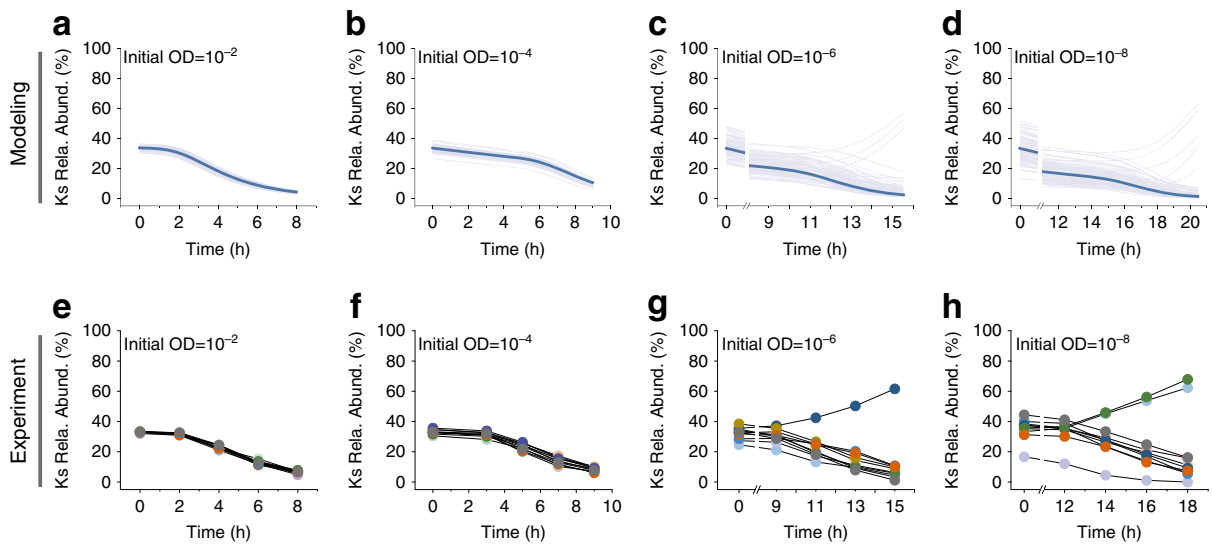

i

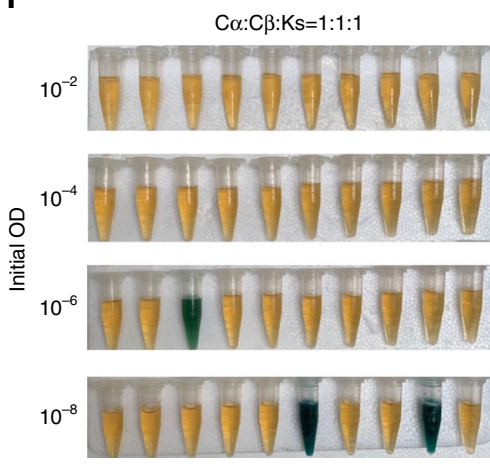

Fig. 4 Divergent ecosystem dynamics directed by cooperation variations from sampling. a-d Model-predicted time courses of Ks abundance in the C $\alpha$ $C \beta-K s$ ecosystem starting with a total initial OD of $10^{-2}$ (a), $10^{-4}$ (b), $10^{-6}$ (c), and $10^{-8}$ (d). For each initial OD, a total of 100 simulation replicates were shown. For comparison, the deterministic dynamics of Ks without sampling-induced variability were displayed with bold lines. $\mathbf{e}-\mathbf{h}$ Experimentally measured population dynamics of $\mathrm{Ks}$ in the $\mathrm{C} \alpha-\mathrm{C} \beta-\mathrm{Ks}$ ecosystem for a total initial OD of $10^{-2}$ (e), $10^{-4}$ (f), $10^{-6}$ ( $\mathbf{g}$ ), and $10^{-8}$ (h). The co-cultures were inoculated at 1:1:1 ratio but different total ODs; for each initial OD, 10 experimental replicates were performed. i Colorimetric assays of the C $\alpha$ - $C \beta$-Ks co-cultures from the experiment in $\mathbf{e}-\mathbf{h}$. Cultures containing strain Ks turned into blue green due to the cleavage of X-Gluc by beta-glucuronidase from Ks; in contrast, those lacking Ks remains the original culture color (yellow). Each test tube contains only the supernatants of the co-culture after X-Gluc treatment. Source Data available in the source data file.

consortium remains largely invariant (Fig. 3g), which was subsequently verified by co-culture experiments (Fig. 3h, Supplementary Fig. 10c, d). In this case, varying initial cooperator partition continued to generate the variation of $\mathrm{ln} G$ production as in the $\mathrm{C} \alpha-\mathrm{C} \beta-\mathrm{Ks}$ consortium (Fig. 2a-c); however, due to Kr's resistance to $\mathrm{lnGG}$, the variation was 'insulated' and not propagated to ecosystem succession, leading to the invariant community structure. These results confirmed that it is indeed the variations of interaction that cause the diversification of ecosystem development.

To examine if the modulation of ecosystem succession by interaction variability is specific to the origin of variation, we investigated the dynamics of the $\mathrm{Ca}-\mathrm{C} \beta-\mathrm{Ks}$ consortium upon fluctuations in sampling, another source of variation we characterized. Mathematically, we used a bottom-up strategy to create a corresponding dynamic model by introducing samplinginduced variations into the previous $\mathrm{Ca}-\mathrm{C} \beta$ co-culture module (Supplementary Equation 4) and Ks monoculture module (Supplementary Equation 5) with experimentally derived parameters (Supplementary Tables 7 and 8) and combining the resulting modules into a single model (Methods). Using the model that encapsulates variations from sampling, we conducted multiple repeats of computational batch fermentations for the consortium starting with the 1:1:1 ratio but different initial ODs. In parallel, we experimentally mixed the strains $C a, C \beta$ and Ks in 1:1:1 ratio to form the consortium, inoculated them into ten replicates at specific initial total ODs $\left(10^{-2}, 10^{-4}, 10^{-6}\right.$, and 10 $-8)$ and measured their population dynamics over time (Methods). Notably, due to the difference in initial conditions, the culturing time and sampling time were altered accordingly to enable a consistent and proper comparison (Supplementary Fig. 12).

Our model predictions and subsequent experiments showed that the Ks fraction consistently declined over time at high initial ODs (e.g., $10^{-2}$ and $10^{-4}$ ) across all replicates (Fig. 4a-f), suggesting that $\mathrm{C} \alpha$ and $\mathrm{C} \beta$ robustly outperformed Ks. Conversely, when the initial OD was low $\left(10^{-6}\right.$ and $\left.10^{-8}\right)$, the consortium exhibited two divergent modes of succession (Fig. 4c-d, g-h): the Ks fraction declined monotonically as in the high initial OD cases; alternatively, it increased over time and dominated the population. Furthermore, comparison of all four cases suggested that the chance of Ks dominance increased with reducing initial OD. To directly visualize such succession outcomes, we further collected the co-cultures at the end of fermentations and performed colorimetric assays by adding X-Gluc to the cocultures (Methods). Because Ks encodes beta-glucuronidase which can produce a clear blue green color, the colors of the treated supernatants (Fig. 4i) reflected the Ks dominance in individual experiments. Linking to the characterization of sampling-induced variations (Fig. 2e, Supplementary Tables 7, 8 ), these results confirmed that increasing sampling-induced variations equally drives the consortium into divergent outcomes. Supporting the statement, we computationally turned off the sampling-induced variations in our mathematical model and found that, without the variations, Ks persistently declined regardless of initial ODs (Fig. 4a-d, bold lines). The theoryexperiment consistency suggested that incorporating variability into ecosystem modeling provides a predictive capacity over community behaviors.

Construction of $\mathrm{pH}$-dependent competition and cooperation. To examine if variability-modulated ecosystem succession is general to different cellular interactions, we designed a new cooperation-competition consortium composed of the cooperators- $\mathrm{C} \alpha$ and $\mathrm{C} \beta$ - and $\mathrm{Kp}$, a strain that opposes $\mathrm{C} \alpha$ and $\mathrm{C} \beta$ and resists their killing in a $\mathrm{pH}$-dependent manner. Using L. lactis MG1363 as the host, the pH-dependent Kp-to-Ca/C $\beta$ inhibition was created by applying a $\mathrm{pH}$-inducible promoter $\mathrm{P}_{774}{ }^{64}$ to control $\operatorname{lcn} A$, the precursor gene of the $\operatorname{lcn} A$ pathway and the constitutive promoters $\mathrm{P}_{1}$ and $\mathrm{P}_{2}$ to drive the rest genes (lce $A$, $\operatorname{lcm} A$, and $l c i A$ ) in the pathway (Fig. 5a). Similarly, the pHdependent resistance to $\mathrm{C} \alpha / \mathrm{C} \beta$-to-Kp inhibition was enabled by using the promoter $\mathrm{P}_{774}$ to drive lagC, the immunity gene of $\mathrm{lcnG}$ co-produced by $\mathrm{Ca}$ and $\mathrm{C} \beta$. Additionally, gusA3 $3^{63}$ was constitutively expressed to enable colorimetric quantification of the strain.

Previous studies showed that the promoter $\mathrm{P}_{774}$ is active when the environmental $\mathrm{pH}$ is below 6.5 but switched to be inactive when above $7^{64}$. Thus, Kp's lcnA production (i.e., inhibition over $\mathrm{C} \alpha$ and $\mathrm{C} \beta$ ) and $\mathrm{LagC}$ production (i.e., resistance to killing by $\mathrm{Ca}$ and $C \beta$ ) are no longer constant but, instead, vary with the environment. Importantly, these strains are all derived from $L$. 
a

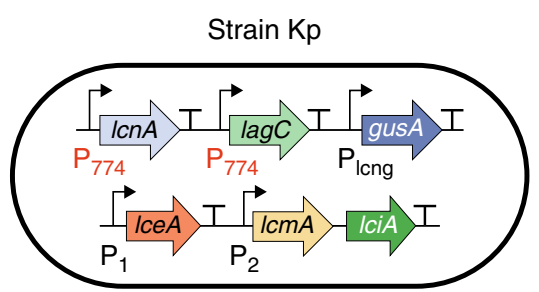

C

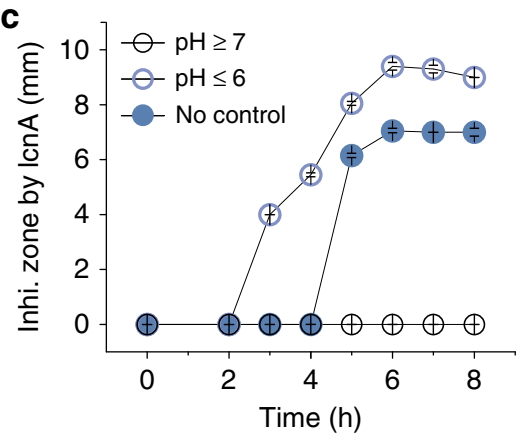

b
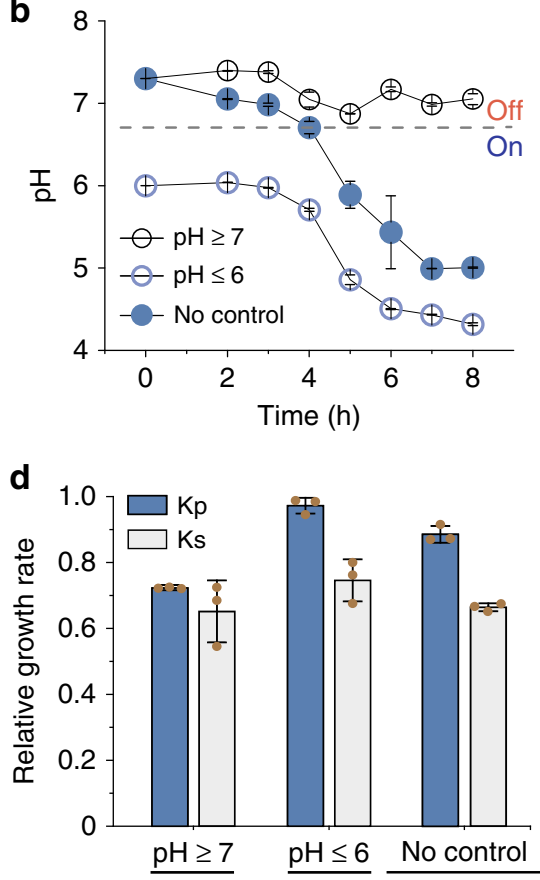

Fig. 5 A synthetic strain whose interactions vary with environmental pH. a Design of the strain $\mathrm{Kp}$. The IcnA precursor gene, IcnA, is driven by the $\mathrm{pH}$ dependent promoter $\mathrm{P}_{774}$ while other IcnA biosynthesis genes IceA, IciA, and IcmA are under the control of native constitutive promoters $\mathrm{P}_{1}$ and $\mathrm{P}_{2}$, leading to a $\mathrm{pH}$-modulated production of IcnA. Meanwhile, lag $\mathrm{C}$, the $\mathrm{IcnG}$ immunity gene, is regulated by the promoter $\mathrm{P}_{774}$, resulting in $\mathrm{pH}$-dependent resistance to IcnG. Additionally, the reporter gene gus $\mathrm{A} 3$ was introduced to $\mathrm{Kp}$ to enable quantification. $\mathbf{b}, \mathbf{c}$ Temporal profiles of extracellular $\mathrm{pH}$ (b) and the sizes of IcnA inhibition zone (c) of $\mathrm{Kp}$ in three $\mathrm{pH}$-defined conditions: $\mathrm{pH} \geq 7, \mathrm{pH} \leq 6$ and no $\mathrm{pH}$ control. d Relative growth rates of $\mathrm{Kp}$ and $\mathrm{Ks}$ in $\mathrm{GM} 17$ media mixed with the supernatant of $\mathrm{C} \alpha-\mathrm{C} \beta$ co-culture normalized by those growing in the media mixed with the $\mathrm{C} \alpha-\mathrm{C} \beta^{\prime}$ supernatant in three $\mathrm{pH}$-defined conditions. Source Data available in the source data file.

lactis which naturally produces a large amount of lactic acid and, thus, can lower the $\mathrm{pH}$ of culture in fermentation, which suggests that both the Kp-to-Ca/C $\beta$ and $C \alpha / C \beta$-to-Kp inhibitions can be highly dynamic even in simple batch fermentation.

To validate the interactions, we grew $\mathrm{Kp}$ monoculture under three settings: $\mathrm{pH} \geq 7, \mathrm{pH} \leq 6$, and no $\mathrm{pH}$ control (Methods) (Fig. 5b and Supplementary Fig. 13). Our results (Fig. 5c and Supplementary Fig. 14) showed that the size of the $\operatorname{lcn} A$ inhibition zones (Methods) remains undetectable during the $\mathrm{pH} \geq 7$ fermentation, suggesting no lcnA production. By contrast, in the $\mathrm{pH} \leq 6$ fermentation, lcnA was detected as early as $3 \mathrm{~h}$ after fermentation and the culture yielded the highest lcnA level. For the case of no $\mathrm{pH}$ control, lcnA was detected after $4 \mathrm{~h}$ of fermentation and eventually accumulated to a medium level. These results confirmed that $\mathrm{lcn} A$ production (Kp-to $\mathrm{Ca} / \mathrm{C} \beta$ inhibition) is highly correlated with the environmental $\mathrm{pH}$. To confirm the $\mathrm{pH}$-dependence of the $\mathrm{Ca} / \mathrm{C} \beta$-to-Kp inhibition, we cultured $\mathrm{Kp}$ and $\mathrm{Ks}$ in $\mathrm{pH}$-defined media mixed with the supernatant of the corresponding $\mathrm{C} \alpha-\mathrm{C} \beta$ or $\mathrm{C} \alpha-\mathrm{C} \beta$ ' co-cultures ( $C \beta^{\prime}$ is a $C \beta$ variant deficient in $\beta$ subunit production), and compared their relative growth rates (Methods, Supplementary Fig. 9e, f and 15). The results (Fig. $5 \mathrm{~d}$ ) showed that Kp grew better than $\mathrm{Ks}$ in all three conditions despite a higher load due to lagC production, demonstrating that $\mathrm{Kp}$ gained a resistance to $\mathrm{lcnG}$. Additionally, the results (Fig. 5d, blue bars) showed that Kp grew best at $\mathrm{pH} \leq 6$ and worst at $\mathrm{pH} \geq 7$, confirming $\mathrm{pH}$ modulates Kp's resistance to lcnG. Equivalently, the results suggested that both the growth of $\mathrm{C} \alpha$ and $\mathrm{C} \beta$ monoculture (Supplementary Fig. 16) and the strength of $\mathrm{C} \alpha-\mathrm{C} \beta$ cooperation are indeed modulated by $\mathrm{pH}$ (Supplementary Fig. 17).

These results further implied that, subject to environmental $\mathrm{pH}$, the $\mathrm{C} \alpha-\mathrm{C} \beta-\mathrm{Kp}$ ecosystem can exhibit multiple modes of interaction. When $\mathrm{pH} \geq 7, \mathrm{Ca}$ and $\mathrm{C} \beta$ cooperatively inhibit $\mathrm{Kp}$ (Fig. 6a); when $\mathrm{pH} \leq 6, \mathrm{Kp}$ inhibits $\mathrm{Ca}$ and $\mathrm{C} \beta$ (Fig. 6b); by contrast, when there is no $\mathrm{pH}$ control, $\mathrm{Ca}$ and $\mathrm{C} \beta$ inhibit $\mathrm{Kp}$ initially but, later, are suppressed by Kp (Fig. 6c).

Dynamics jointly regulated by multiple interaction variations. To illustrate how the Ca-C $\beta-\mathrm{Kp}$ consortium evolves upon both cooperation and competition variations, we assembled a dynamic community model (Supplementary Equation 17) from the bottom up (Methods). Then we used the model to explore the succession of the consortium when its interaction strengths vary due to simultaneous $\mathrm{pH}$ and cooperator partition alterations. In parallel, we performed $\mathrm{Ca}-\mathrm{C} \beta-\mathrm{Kp}$ co-culture experiments under the conditions identical to the computational test (Methods).

For the consortium starting from a fixed initial $(\mathrm{C} \alpha+\mathrm{C} \beta): \mathrm{Kp}$ ratio (2:1), the model predicted and subsequent experiments confirmed that, when $\mathrm{pH}$ was controlled above $7, \mathrm{Kp}$ abundance declined gradually over fermentation for different initial Ca:C $\beta$ ratios but reached to the lowest at 1:1 (Fig. 6d, g). In contrast, when $\mathrm{pH}$ was below $6, \mathrm{Kp}$ became increasingly dominant over time for all $\mathrm{Ca}: \mathrm{C} \beta$ ratios but augmented the most at unbalanced cases (30:1 and 1:30) (Fig. 6e, h). When there was no $\mathrm{pH}$ control, the consortium succession displayed two distinct patterns: At unbalanced ratios, $\mathrm{Kp}$ evolved to be dominant as the case of $\mathrm{pH} \leq$ 6 but, at close ratios, Kp abundance declined over time as the $\mathrm{pH} \geq 7$ case (Fig. 6f, i).

Although seemingly diversified, these successions can be elucidated by considering the multiple interaction variations caused by $\mathrm{pH}$ modulation and labor partition. First, the systematic shift of the Kp abundance from consistent decrease (Fig. 6d, g) to consistent increase (Fig. 6e, h) and divergent 
a

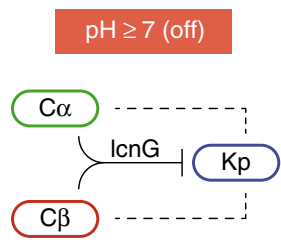

d

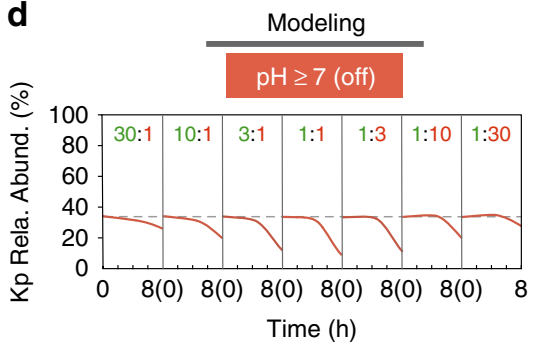

g

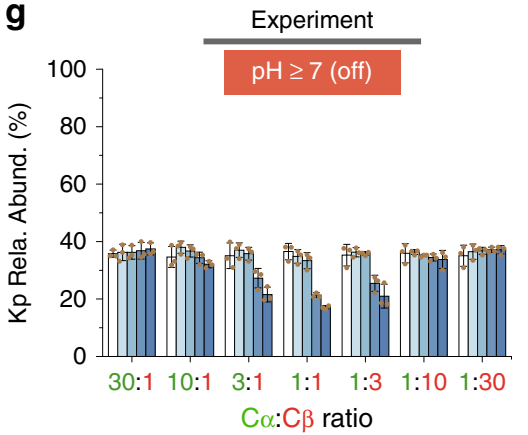

b

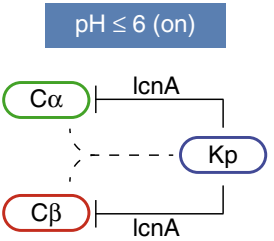

e

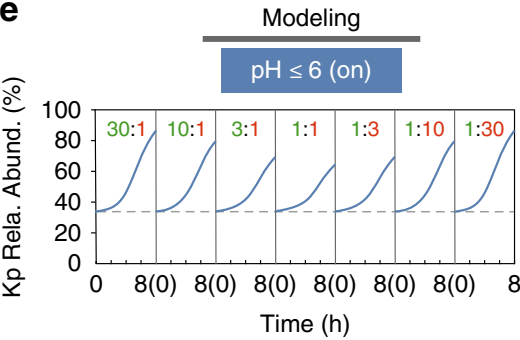

h
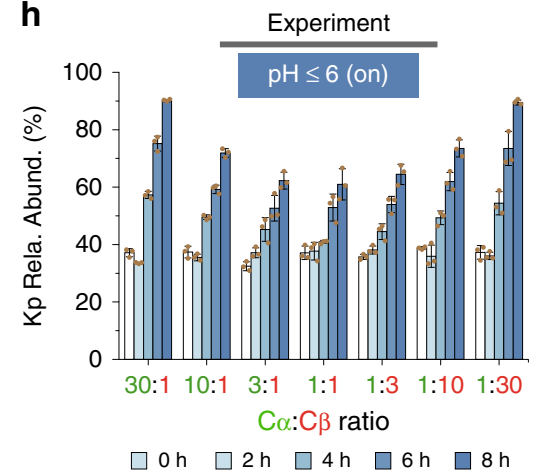

c

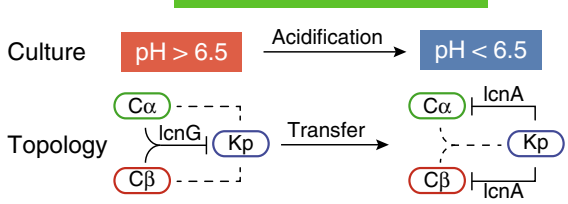

f

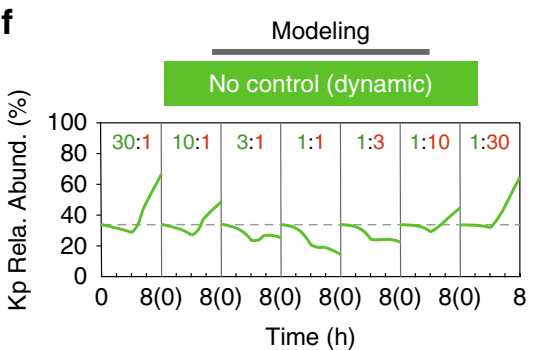

i
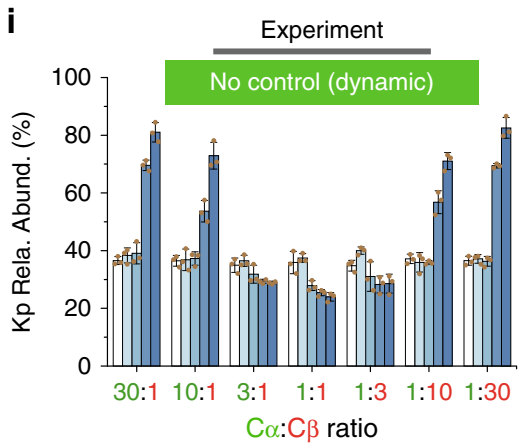

Fig. 6 Ecosystem dynamics jointly regulated by cooperation variations and competition variations. a-c Interaction topology of the $C \alpha-C \beta-K p$ ecosystem in different $\mathrm{pH}$ settings. At $\mathrm{pH} \geq 7$ (a), the promoter $\mathrm{P}_{774}$ remains inactive (i.e., 'Off' state), which abolishes $\mathrm{Kp}$-to- $\mathrm{C} \alpha / \mathrm{C} \beta$ inhibition while allowing $\mathrm{C} \alpha$ and $\mathrm{C} \beta$ to suppress $\mathrm{Kp}$. At $\mathrm{pH} \leq 6$ (b), the promoter $\mathrm{P}_{774}$ is active (i.e., 'On' state), leading to effective $\mathrm{Kp}$-to- $\mathrm{C} \alpha / \mathrm{C} \beta$ inhibition but diminished $\mathrm{C} \alpha / \mathrm{C} \beta$-to-Kp suppression. Where there is no $\mathrm{pH}$ control (c), the promoter dynamically transits from inactive to active (i.e., 'Dynamic' state) with $\mathrm{pH}$ reduction upon fermentation. As a result, the interaction network switches from $\mathrm{C} \alpha / \mathrm{C} \beta$ killing Kp to Kp killing $\mathrm{C} \alpha / \mathrm{C} \beta$. d-i Model-predicted (d-f) and experimentally measured ( $\mathbf{g}-\mathbf{i})$ temporal evolution of $\mathrm{Kp}$ abundance in three $\mathrm{pH}$-defined settings and under varied initial $\mathrm{C} \alpha: \mathrm{C} \beta$ partitions. Although $\mathrm{C} \alpha: \mathrm{C} \beta$ ratio was varied from 30:1 to 1:30, the total $C \alpha$ and $C \beta$ concentration was kept at 2:1 ratio with the Kp concentration. Source Data available in the source data file.

development (Fig. 6f, i) originated from the $\mathrm{pH}$-induced variation of the interactions: At $\mathrm{pH} \geq 7, \mathrm{Kp}$-to- $\mathrm{Ca} / \mathrm{C} \beta$ inhibition was abolished but $\mathrm{C} \alpha / \mathrm{C} \beta$-to-Kp inhibition remained potent; at $\mathrm{pH} \leq$ 6, Kp-to-C $\alpha / \mathrm{C} \beta$ inhibition became effective (Supplementary Fig. 18) but $\mathrm{C} \alpha / \mathrm{C} \beta$-to-Kp inhibition was significantly reduced; when there was no $\mathrm{pH}$ control, $\mathrm{C} \alpha$ and $\mathrm{C} \beta$ inhibited $\mathrm{Kp}$ at beginning but were later suppressed by Kp (Fig. 6a-c). Second, within a single $\mathrm{pH}$ setting, the final $\mathrm{Kp}$ abundance was lower at close $\mathrm{Ca}: \mathrm{C} \beta$ partitions (e.g. 1:1) than at imbalanced (e.g., 30:1 and 1:30) because $C \alpha$ and $C \beta$ had a stronger lcnG productivity when their partitions are close (Supplementary Fig. 17). These results showed that for ecosystems containing multiple variable interactions, at least for those we tested, their succession is determined jointly by all of the variations but not by any one of them. Of note, the divergent dynamics in the absence of $\mathrm{pH}$ control (Fig. 6f, i) exemplified the superposition of $\mathrm{pH}$ and labor partition effects: At imbalanced $C a: C \beta$ partitions, $C \alpha$ and $C \beta$ had the potential to kill $\mathrm{Kp}$ at beginning but their lcnG yield was too low; later, $\mathrm{Kp}$ gained the $\mathrm{lnG}$ resistance and further secreted lcnA to kill $\mathrm{Ca}$ and $\mathrm{C} \beta$, leading to the monotonic increase of $\mathrm{Kp}$ abundance. In contrast, at the close partitions, $C \alpha$ and $C \beta$ produced significant lcnG to efficiently inhibit Kp during the initial fermentation and the lcnG remained in the culture continued to suppress $\mathrm{Kp}$ even though the interaction topology was later altered.
To further demonstrate this finding, we conducted additional assays for the consortium by varying $\mathrm{pH}$ and initial total OD. Here, the model and the experimental setups were the same as previous except for the initial conditions (Methods). Accordingly, the sources of variation became $\mathrm{pH}$ modulation and random sampling. Our results showed that, at $\mathrm{pH} \geq 7$, the $\mathrm{Kp}$ abundance consistently reduced at high initial ODs (Fig. $7 \mathrm{a}-\mathrm{f}$ ), owing to the cooperative inhibition of $\mathrm{C} \alpha$ and $\mathrm{C} \beta$ to Kp; However, at low initial ODs, it could also remain largely invariant in some replicates (Fig. $7 \mathrm{c}-\mathrm{d}, \mathrm{g}-\mathrm{h}$ ) since increasing variations at random sampling abolished the cooperation (Fig. 2e-f). By contrast, in the absence of sampling-induced variations, the $\mathrm{Kp}$ abundance always declined regardless of initial ODs (Fig. 7a-d, bold lines). At $\mathrm{pH} \leq 6, \mathrm{Kp}$ became increasingly dominant regardless of initial ODs (Fig. 7i-l, $\mathrm{m}-\mathrm{p}$ ), because Kp constitutively suppressed $\mathrm{Ca}$ and $C \beta$ and such a suppression was not affected by the fluctuations of $C \alpha: C \beta$ ratios. These results are consistent with the ecosystem succession when sampling-induced variations were eliminated (Fig. 7i-l, bold lines). When there was no $\mathrm{pH}$ control, Kp declined minorly to a plateau at high initial ODs (Fig. 7q-r, $u-v)$, attributed to the factors that $C a$ and $C \beta$ collaborated to suppress $\mathrm{Kp}$ initially but were later suppressed by $\mathrm{Kp}$ due to $\mathrm{pH}$ reduction. At low initial ODs, it declined as the high initial OD case or diverged to be dominant (Fig. $7 \mathrm{~s}-\mathrm{t}, \mathrm{w}-\mathrm{x}$ ) because increasing randomness diminished the $\mathrm{C} \alpha / \mathrm{C} \beta$-to-Kp inhibition at 

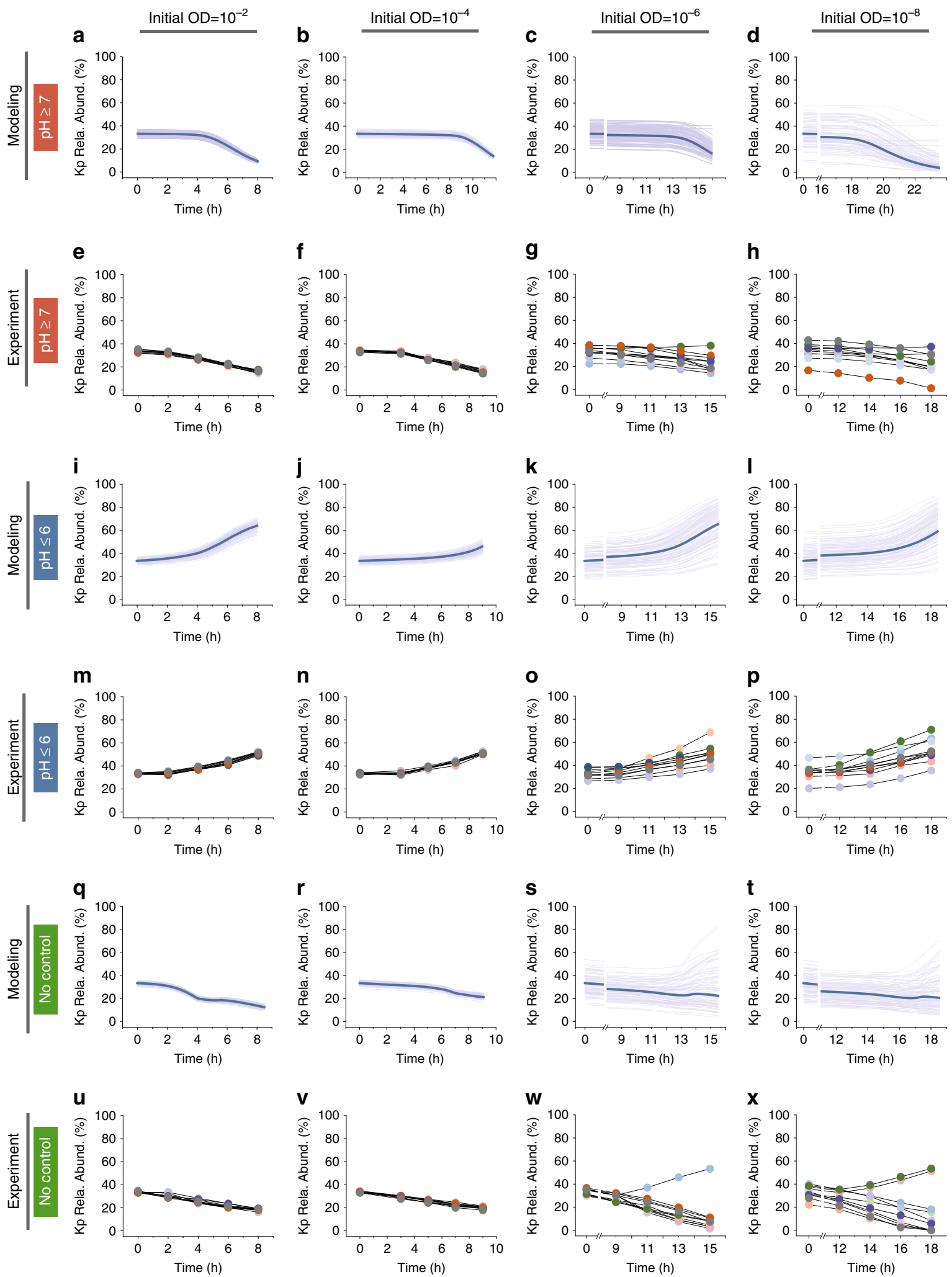

Fig. 7 Model-predicted and experimentally measured succession of the $\mathbf{C} \alpha-\mathbf{C} \beta-\mathrm{Kp}$ ecosystem for varied pH conditions and initial densities. a-h Predicted (a-d) and experimentally measured (e-h) time courses of $\mathrm{Kp}$ abundance in the $\mathrm{C} \alpha-\mathrm{C} \beta$-Kp consortium when $\mathrm{pH} \geq 7$. $\mathbf{i}-\mathbf{p}$ Predicted $(\mathbf{i}-\mathbf{I})$ and measured ( $\mathbf{m}-\mathbf{p}$ ) time courses of $\mathrm{Kp}$ abundance in the $\mathrm{C} \alpha-\mathrm{C} \beta-\mathrm{Kp}$ consortium when $\mathrm{pH} \leq 6 . \mathbf{q}-\mathbf{x}$ Predicted (q-t) and measured (u-x) time courses of Kp abundance in the $\mathrm{C} \alpha-\mathrm{C} \beta-\mathrm{Kp}$ consortium when there is no $\mathrm{pH}$ control. For both model predictions and experimental measurements, the co-culture was inoculated at 1:1:1 ratio but the initial OD was varied from $10^{-2}$ (first column) to $10^{-4}$ (second column), $10^{-6}$ (third column) and $10^{-8}$ (fourth column). For each condition, a total of 100 simulation replicates and 10 experimental replicates were performed. For comparison, the deterministic dynamics of Kp without sampling-induced variability were displayed with bold lines in $\mathbf{a}-\mathbf{d}, \mathbf{i}-\mathbf{I}$, and $\mathbf{q}-\mathbf{t}$. Source Data available in the source data file. 
the beginning but did not affect $\mathrm{Kp}$-to- $\mathrm{C} \alpha / \mathrm{C} \beta$ suppression later. For comparison, in the absence of variation, there was no $\mathrm{Kp}$ dominance under all initial conditions (Fig. $7 \mathrm{q}-\mathrm{t}$, bold lines). These results demonstrated again that it is the joint regulation from multiple interaction variations that determines the dynamics of the ecosystems.

To quantitatively evaluate the capacity of the variabilityincorporated modeling scheme, we calculated relative errors, defined as the differences between simulation and experimental results divided by experimental measures, for all of the simulations and experimental data above. The results (Supplementary Fig. 19) showed that, for most of the comparisons, the simulations agree quantitatively with experimental measures with the mean absolute relative errors (MARE) falling within the range of $(0,0.2)$ (panels a-d, g). For a subset of the cases, the models have larger relative errors but yet qualitatively agree well with the experimental findings (panels e, f, h). The encouraging agreements between the simulations and experiments demonstrated that incorporating interaction variability into ecosystem modeling is a promising strategy for quantitative and predictive understanding of complex community behaviors. Meanwhile, the discrepancies in certain cases suggest that the current models may need to consider additional processes involved in the experimental ecosystems, such as the nonlinearity observed during parameter fitting in Supplementary Fig. 8, in order to achieve a better modeling-experiment agreement.

\section{Discussion}

Microbial interactions are often modeled invariant ${ }^{20}$; however, in nature, they constantly fluctuate over time and such fluctuations in strength are shown to be profound to ecosystem behaviors ${ }^{17-}$ 32. Using synthetic microbial consortia as simple and reliable platforms, we showed that increasing variations of interaction diversifies an ecosystem's succession into distinct outcomes. We also showed that, when there are multiple variable interactions ${ }^{18,19,25}$, these variations collectively, but not a single one, regulate the behaviors of a community. Together, our results established interaction variability as a critical modulator of ecosystem behaviors.

Our synthetic ecosystems are relatively simple as they contain only a competition and a cooperation. Yet, their dynamics can be dramatically modulated by the variations of interaction. In native ecologies, there are significantly more cellular interactions and more microbial and environmental factors that modulate cellular interactions. We thus speculate that interaction variability is not specific to our synthetic systems and potentially a universal determinant for microbial ecosystem succession.

Searching for assembly rules has been invaluable to our understanding of community organization ${ }^{65-67}$. In our study, we observed the diversification of ecosystem succession with increasing variations, which illustrates the intrinsic complexity and context-dependence nature of community behaviors. This finding implies that, in certain scenarios, qualitative rules may not be sufficient to specify the assembly of a community; instead, quantitative and systematic characterizations are needed. The result might also partially explain the current difficulty in predicting microbial ecosystem behaviors, an existing key challenge in microbial ecology 4 .

Lastly, our study shows encouraging consistence between experiment and mathematical modeling, which illustrates the promise of variability-cast ecosystem modeling for bottom-up predictions of the structure and dynamics of microbial ecosystems. Together, this work provides fundamental insights into the organization of microbial communities and also the de novo engineering of microbial consortia for various biotechnological applications.

\section{Methods}

Strain and growth conditions. All strains are derived from L. lactis MG1363 and grown at $30^{\circ} \mathrm{C}$ in $\mathrm{M} 17$ broth supplemented with $0.5 \%(\mathrm{w} / \mathrm{v})$ glucose and $5 \mu \mathrm{g} \mathrm{mL}^{-1}$ of chloramphenicol $(\mathrm{GM} 17 / \mathrm{Cm})$. Tween 80 was added at a final concentration of $0.1 \%(\mathrm{v} / \mathrm{v})$ when necessary. Cell cultures are adjusted with $2 \mathrm{M} \mathrm{NaOH}$ solution every hour to maintain $\mathrm{pH}$ above 7 . To achieve $\mathrm{pH} \leq 6$, cell cultures are adjusted by $1 \mathrm{M} \mathrm{HCl}$ every hour. Strains used in this study are described in Supplementary Table 1.

Plasmid construction. All plasmids used in this study were developed from a $L$. lactis-E. coli shuttle vector, pleiss-Nuc ${ }^{68}$, and described in Supplementary Table 1. Oligos for plasmid construction are listed in Supplementary Table 2. To generate the plasmid pleiss-lcnG for lactococcin $\mathrm{G}$ production, a 5-kb fragment of lcnG gene cluster including $\operatorname{lag} A, \operatorname{lag} B, \operatorname{lag} C, \operatorname{lag} D$, and $\operatorname{lag} E$ was amplified from the genome of L. lactis LMG 2081 using primers of lcnG-F and lcnG-R ${ }^{53}$, and subsequently assembled with a fragment of pleiss-Nuc amplified with primers $P_{g}-F$ and $P_{g}-R$ using Gibson assembly. The plasmid pleiss-lenG was then transformed into L. lactis MG1363 to obtain the lcnG producing strain Caß. To construct lcnG subunit expression plasmids pleiss-C $\alpha_{0}$ and pleiss-C $\beta_{0}$, the $\alpha$ or $\beta$ coding gene was deleted from the plasmid pleiss-lenG by reverse PCR and Gibson assembly using two pairs of primers: $C \alpha_{0}-\mathrm{F} / \mathrm{Ca}_{0}-\mathrm{R}$ and $\mathrm{C} \beta_{0}-\mathrm{F} / \mathrm{C} \beta_{0}-\mathrm{R}$. The resulting plasmids were transformed into L. lactis MG1363 to generate strains $C \alpha_{0}$ and $C \beta_{0}$. To enable screening and counting of cells with different subunits, a gfp or rfp reporter gene, yemGFP or $m$ Cherry was introduced using primers $\mathrm{P}_{c \alpha}-\mathrm{F} / \mathrm{P}_{\mathrm{ca}}-\mathrm{R}$ and $\mathrm{Ca}-\mathrm{F} / \mathrm{Ca}-\mathrm{R}$, generating the plasmids pleiss-C $\alpha$ and pleiss-C $\beta$. These plasmids were subsequently transformed into L. lactis MG1363 to construct the reporter version of $\alpha$ and $\beta$ peptide producer $\mathrm{C} \alpha$ and $C \beta$. As a control, plasmid pleiss-C $\beta$ ' was generated by deleting the $\beta$ precursor gene $\operatorname{lag} B$ from the plasmid pleiss- $C \beta$ with primers $\mathrm{P}_{C \beta}, \mathrm{F}, \mathrm{P}_{\mathrm{C} \beta}, \mathrm{F}, \mathrm{C} \beta^{\prime}-\mathrm{F}$ and $C \beta^{\prime}-\mathrm{R}$. The resulting plasmid was then transformed into L. lactis MG1363 to get a $\beta$-free variant $C \beta$ '. To increase the productivity of $\alpha$ peptide in $\mathrm{Ca}_{0}$, an additional copy of expression cassette of $\alpha$ under the control of $\mathrm{P}_{4}$ promoter was inserted to pleiss- $\mathrm{Ca}_{0}$ using primers $\mathrm{P}_{\mathrm{ca} 0-\mathrm{P} 4 \alpha^{-}}-\mathrm{F} / \mathrm{P}_{\mathrm{ca} 0-\mathrm{P} 4 \mathrm{a}}-\mathrm{R}$ and $\mathrm{Ca}_{0}-\mathrm{P}_{4 \mathrm{a}}-\mathrm{F} / \mathrm{Ca}_{0}-\mathrm{P}_{4 \mathrm{a}}$ $\mathrm{R}^{69}$, generating the variant $C \alpha_{0 \mathrm{v} 2}$. $C \beta_{0 \mathrm{v} 1 / 3}$ and $C \beta_{0 \mathrm{v} 1 / 5}$, with approximately $1 / 3$ and $1 / 5$ of the productivity of $C \beta_{0}$ respectively, were created through inserting simple short repeat sequence $(\mathrm{AT})_{\mathrm{n}}$ into the spacer region of ribosome binding site of $\beta$ peptide to weaken the translational initiation rate with primers $\mathrm{P}_{\mathrm{C} \beta 0 \mathrm{v} 1 / 3}-\mathrm{F} / \mathrm{P}_{\mathrm{C} \beta 0 \mathrm{v} 1 / 3^{-}}$ $\mathrm{R}, \mathrm{C} \beta_{0 \mathrm{v} 1 / 3}-\mathrm{F} / \mathrm{C} \beta_{0 \mathrm{v} 1 / 3}-\mathrm{R}, \mathrm{P}_{\mathrm{C} \beta 0 \mathrm{v} 1 / 5}-\mathrm{F} / \mathrm{P}_{\mathrm{C} \beta 0 \mathrm{v} 1 / 5}-\mathrm{R}, \mathrm{C} \beta_{0 \mathrm{v} 1 / 5}-\mathrm{F} / \mathrm{C} \beta_{0 \mathrm{v} 1 / 5}-\mathrm{R}^{70}$. The lactococcin A producing plasmid pleiss-lcnA was constructed by assembling the lcnA gene cluster from the plasmids pFI2396 and pFI2148 with pleiss-Nuc vector ${ }^{62}$. To simplify the detection of the lcnA-producing strain, a reporter gene gusA 3 was amplified from the plasmid pTRK $892^{63}$ and then inserted into pleiss-lcnA using primers $\mathrm{P}_{\text {lcnA-gusA3 }}-\mathrm{F} / \mathrm{P}_{\text {lcnA-gusA3 }}-\mathrm{R}$ and lcnA-gusA3-F/lcnA-gusA3-R. The resulting plasmid pleiss-lcnA-gusA3 was transformed into L. lactis MG1363 to generate the lcnA-producing strain Ks. Kr, as a control strain for Ks with immunity to lcnG and a gusA 3 reporter, was created by assembling the immunity gene lag $C$ of $l \mathrm{cnG}$ and GusA3 using the primers of $\mathrm{P}_{\mathrm{IG}-\mathrm{GusA} 3}-\mathrm{F} / \mathrm{P}_{\mathrm{IG}-\mathrm{GusA3}}-\mathrm{R}$ and GusA3-F/GusA3-R. To create the strain with $\mathrm{pH}$-dependent $\mathrm{lcnA}$ production and $\mathrm{lcnG}$ resistance, plasmid pleiss- $\mathrm{P}_{774}$-lcnA-gusA3 was firstly created by replacing the lcnA's native promoter $\mathrm{P}_{\text {lcna }}$ of the plasmid pleiss-lcnA-gusA3 with a $\mathrm{pH}$-inducible promoter $\mathrm{P}_{774}{ }^{64}$. Subsequently, pleiss- $\mathrm{P}_{774}$-lcnA-gusA3 was assembled with the fragment of $\mathrm{P}_{774}$ lagC using two pairs of primers: $\mathrm{P}_{\mathrm{p} 774-\mathrm{lcn} A}-\mathrm{F}$ and $\mathrm{P}_{\mathrm{p} 774-\mathrm{lcnA}}-\mathrm{R}, \mathrm{P}_{774}-\mathrm{lcn} \mathrm{A}-\mathrm{F}$ and $\mathrm{P}_{774}$-lcnA-R; $\mathrm{P}_{\mathrm{p} 774-\operatorname{lagC}}-\mathrm{F}$ and $\mathrm{P}_{\mathrm{p} 774-\operatorname{lagC}}-\mathrm{R}, \mathrm{P}_{774}-\mathrm{lagC}-\mathrm{F}$ and $\mathrm{P}_{774}-\mathrm{lagC}-\mathrm{R}$, resulting in the final plasmid pleiss- $\mathrm{P}_{774}-\mathrm{lcnA}_{\mathrm{A}}-\mathrm{P}_{774}$-lagC-gusA3. The final plasmid was then transformed into L. lactis MG1363 to generate the strain Kp.

Measurement of IcnG productivity. The agar diffusion assay was performed using a protocol adapted from a previous study ${ }^{55}$. Specifically, cultures of $l \mathrm{cnG}$ producing cells were grown in $\mathrm{GM} 17 / \mathrm{Cm} / \mathrm{Tween}$ broth at $30^{\circ} \mathrm{C}$ overnight under corresponding culture conditions. The overnight cultures were inoculated in fresh media at 1:50 dilution and grown to the early stationary phase. Supernatants were obtained by centrifuging at $10,000 \times g$ for $10 \mathrm{~min}$. Then, $30 \mu \mathrm{L}$ samples were added into the wells in a double-layer agar $(15 \mathrm{~mL}$ of $\mathrm{GM} 17 / \mathrm{Cm} / \mathrm{T}$ ween with $0.75 \%$ agar for each layer) in which the bottom layer was seeded with $50 \mu \mathrm{L}$ of overnight culture of inducer strain L. lactis NZ9000/pleiss-Nuc. After incubation at $30^{\circ} \mathrm{C}$ for $8 \mathrm{~h}$, the inhibition zones were characterized by the blank circles around wells. To establish a standard curve of relative $\mathrm{lcnG}$ concentration for quantitatively evaluating samples, the concentration of lcnG in the supernatant of Caß culture at the early stationary phase is defined as $100 \%$. And then the cell-free supernatant of Ca $\beta$ culture was diluted with fresh GM17/Cm/Tween media to the relative $1 \mathrm{cnG}$ concentrations of $90 \%, 80 \%, 70 \%, 60 \%, 50 \%, 40 \%, 30 \%, 20 \%, 10 \%, 5 \%, 2 \%$, and $1 \%$. Next, $30 \mu \mathrm{L}$ of samples were added into the wells in the top layer agar. After incubation at $30^{\circ} \mathrm{C}$ for $8 \mathrm{~h}$, the inhibition zones emerged and a standard curve was drawn by measuring the diameters of inhibition zones produced by different relative concentrations of $\mathrm{lcnG}$. Using this curve, the relative concentrations of lcnG from tested samples were estimated. 
Characterization of variations from labor partition. Monocultures of $\mathrm{C} \alpha$ and $\mathrm{C} \beta$ $\left(\mathrm{Ca}_{0}\right.$ and $\left.\mathrm{C} \beta_{0}\right)$ were inoculated in $\mathrm{GM} 17 / \mathrm{Cm} / \mathrm{T}$ ween liquid medium at $30^{\circ} \mathrm{C}$ overnight. Then the optical density at $600 \mathrm{~nm}(\mathrm{OD})$ of monocultures were measured and the co-cultures of $C \alpha$ and $C \beta\left(C a_{0}\right.$ and $\left.C \beta_{0}\right)$ were mixed at a variety of ratios $(30: 1,10: 1,3: 1,1: 1,1: 3,1: 10,1: 30)$ with a start total $\mathrm{OD}_{600}$ of $10^{-2}$. After $8 \mathrm{~h}$, the co-culture cells were centrifuged at $10,000 \times g$ for $10 \mathrm{~min}$ to obtain the coculture supernatants. To test the cooperative strength in supernatant mixtures, overnight monocultures were firstly transformed to fresh GM17/Cm/Tween broth at the initial $\mathrm{OD}_{600}$ of $10^{-2}$ individually. Then supernatants were extracted after growth for $8 \mathrm{~h}$ by centrifuging and mixed at different ratios as mentioned above. Finally, $30 \mu \mathrm{L}$ of samples were used to determine the activity of lcnG by agar diffusion assays to examine the variability of cooperation in co-culture supernatants and monoculture supernatant mixtures. Additionally, to evaluate the role of subunit stoichiometric ratio in cooperation variation, monoculture supernatants were also prepared from $\mathrm{Ca}_{0}, \mathrm{Ca}_{0 \mathrm{v} 2}, \mathrm{C}_{0 \mathrm{v} 1 / 3}$, and $\mathrm{C} \beta_{0 \mathrm{v} 1 / 5}$ as described above and mixed at ratios from 30:1, 10:1, 3:1, 1:1, 1:3, 1:5, 1:6, 1:10 to 1:30. Among them, ratios of 1:5 and 1:6 were used for determining the optimal cooperativity between $\mathrm{Ca}_{0}$ and $\mathrm{C} \beta_{0 \mathrm{v} 1 / 5}\left(\mathrm{Ca}_{0 \mathrm{vv} 2}\right.$ and $\left.\mathrm{C} \beta_{0 \mathrm{vv} 1 / 3}\right)$. Then, activities of $\mathrm{lnG}$ in these combinations were determined by agar diffusion assay. Similarly, to measure the cooperative inhibition from $\mathrm{C} \alpha$ and $\mathrm{C} \beta$ to $\mathrm{Ks}(\mathrm{Kp})$, three steps were involved. First, starting media were prepared by mixing 2 -fold concentrated GM17/Cm/Tween medium with equal volume of supernatants from $\mathrm{C} \alpha-\mathrm{C} \beta$ co-culture at the early stationary phase that were filtered with sterile $0.22 \mu \mathrm{m}$ filter and adjusted to necessary $\mathrm{pH}$. Second, overnight Ks $(\mathrm{Kp})$ monoculture was inoculated with an initial OD of $10^{-2}$ to the starting media. Third, the relative abundances of Ks were measured with fluorescence microscope from the starting to $8 \mathrm{~h}$ of incubation, during which $\mathrm{pH}$ was controlled properly if needed. For comparison, starting media were also prepared by mixing 2 -fold concentrated $\mathrm{GM} 17 / \mathrm{Cm} /$ Tween medium with equal volume of supernatants of $\mathrm{C} \alpha-\mathrm{C} \beta^{\prime}$ co-culture for culturing and measuring $\mathrm{Ks}(\mathrm{Kp})$. Each experimental condition has three replicates $(n=3)$.

Determination of the maximum dilution rate. To determine the minimum $\mathrm{OD}_{600}$ that is required for cell growth in a fresh medium, monocultures of $C \alpha_{0}, C \beta_{0}, \mathrm{Ca}$, $C \beta$ and Ks were serially diluted to an $\mathrm{OD}_{600}$ of $10^{-2}$ to $10^{-13}$ at 1:10 dilution and the survival rate of each $\mathrm{OD}_{600}$ was calculated by counting the growing cultures in twenty replicates of each $\mathrm{OD}_{600}$. After incubation at $30^{\circ} \mathrm{C}$ for $24 \mathrm{~h}$, the tubes with cell growth were counted to calculate the survival rate of different initial $\mathrm{OD}_{600}$. Our experiment (Supplementary Fig. 5) showed that $10^{-8}$ is the minimum $\mathrm{OD}_{600}$ with a $100 \%$ survival rate for almost all strains. An inoculation with an initial $\mathrm{OD}_{600}$ lower than $10^{-8}$ could result in failure in growth and would disturb the studies of small number fluctuations in the community. Therefore, the initial $\mathrm{OD}_{600}$ after dilution in the serial dilution experiments should be higher than $10^{-8}$.

\section{Characterization of cooperation variations from sampling. To examine the} effect of sampling on the variability of $\mathrm{C} \alpha-\mathrm{C} \beta$ cooperation, overnight cultures of $\mathrm{Ca}$ and $\mathrm{C} \beta$ were washed twice with sterile $\mathrm{PBS}$ buffer $(\mathrm{pH}=7)$ and re-suspended in PBS buffer. Then, the $C \alpha$ and $C \beta$ suspensions were adjusted to an $\mathrm{OD}_{600}$ of 1.0 with PBS buffer, and mixed together at 1:1 ratio. The resulting suspension was used as a start culture and diluted to the $\mathrm{OD}_{600}$ of $10^{-2}, 10^{-4}, 10^{-6}$ and $10^{-8}$ through serial 1:10 dilutions with PBS buffer. The total volume of a start culture was set at $5 \mathrm{~mL}$. After being prepared, all samples were centrifuged at $10,000 \times \mathrm{g}$ for $15 \mathrm{~min}$ to remove supernatants, and $5 \mathrm{~mL}$ of fresh GM17/Cm media were subsequently added for cell growth. At the end of incubation, the supernatants were obtained. Subsequently, inhibition zone assays were conducted to determine the strength of cooperation. As cultures with different initial ODs require different incubation times, for each initial condition we chose sampling time based on the corresponding growth profile so that the ODs at each time point are comparable across the samples (Supplementary Fig. 12). Such samplings enable a consistent and proper comparison. Ten trials were performed for each initial condition.

Three-strain cooperator-varying experiments. The initial total $\mathrm{OD}_{600}$ of three strains $\mathrm{C} \alpha, \mathrm{C} \beta$ and $\mathrm{Ks}$ ( $\mathrm{Kr}$ or $\mathrm{Kp}$ ) was set at $10^{-2}$. The start abundance of $\mathrm{Ks}(\mathrm{Kr}$ or Kp) was fixed at $33.3 \%$ in the population but the ratios of $C \alpha$ and $C \beta$ were set at 30:1, 10:1, 3:1, 1:1, 1:3, 1:10, and 1:30. During incubation, samples were taken every two hours for measuring their ODs and the relative numbers of green $(\mathrm{C} \alpha)$, red $(C \beta)$, and non-fluorescent cells were counted under an AMG EVOS FL fluorescence microscope using green, red and bright field channels. Notably, for the $\mathrm{pH}-$ controlled three-strain experiments, the overnight culture of $\mathrm{Kp}$ was washed twice with sterile PBS buffer $(\mathrm{pH}=7)$ and then inoculated with an OD of $10^{-2}$ into fresh medium with proper different $\mathrm{pH}$ controls $(\mathrm{pH} \geq 7, \mathrm{pH} \leq 6$ and no $\mathrm{pH}$ control). During the course of fermentation, culture samples were collected every hour to determine environmental $\mathrm{pH}$ using $\mathrm{pH}$ meter and Kp's lcnA productivity using the inhibition zone assay. Each culture condition has three replicates $(n=3)$. The $\mathrm{pH}$ and inhibition zones were calculated as mean \pm s.d.

Three-strain sampling experiments. For the three-strain ecosystem of $C a, C \beta$, and $\mathrm{Ks}(\mathrm{Kp}), \mathrm{C} \alpha$ and $\mathrm{C} \beta$ PBS suspensions were individually adjusted to $\mathrm{OD}_{600}$ of 1 and then mixed at 1:1 ratio; Ks (Kp) PBS suspension was also adjusted to $\mathrm{OD}_{600}$ of
1. Subsequently, both the $\mathrm{C} \alpha-\mathrm{C} \beta$ mixture and $\mathrm{Ks}(\mathrm{Kp})$ were diluted to the $\mathrm{OD}_{600}$ of $10^{-2}, 10^{-4}, 10^{-6}$, and $10^{-8}$ with PBS buffer in 1:10 dilution fold. At each concentration, the $\mathrm{C} \alpha-\mathrm{C} \beta$ mixture and $\mathrm{Ks}(\mathrm{Kp})$ monoculture suspensions were mixed at a 2:1 ratio to reach a final ratio of 1:1:1 for $C a, C \beta$ and $K s(\mathrm{Kp})$. The total volumes of both monoculture and co-culture suspensions were set at $5 \mathrm{~mL}$. The prepared samples were centrifuged at $10,000 \times g$ for $15 \mathrm{~min}$ to remove supernatants, and $5 \mathrm{~mL}$ of fresh GM17/Cm media were subsequently added for cell growth. The co-cultures were then incubated at $30^{\circ} \mathrm{C}$ and propagated for $8\left(10^{-2}\right), 9\left(10^{-4}\right), 14$ $\left(10^{-6}\right)$ and $20\left(10^{-8}\right)$ hours which was needed for entering the early stationary phase. To consistently determine cell numbers in different initial ODs (e.g., $10^{-2}$ $\left.10^{-4}, 10^{-6}, 10^{-8}\right)$, the initial relative abundances of $\mathrm{C} \alpha, \mathrm{C} \beta$ and $\mathrm{Ks}(\mathrm{Kp})$ were measured by colony forming unit (CFU) counting instead of flow cytometry, because flow cytometry typically requires a minimum cell number $\left(10^{4}\right.$ cells per $\mathrm{mL}$ ) in samples. For the samples with an initial $\mathrm{OD}_{600}$ of $10^{-2}$ or $10^{-4}$, the mixed suspensions were diluted to around $\mathrm{OD}_{600}$ of $5 \times 10^{-6}$ with sterile PBS buffer and 1 $\mathrm{mL}$ of the diluted suspension was plated on the GM17/Cm plate supplemented with X-Gluc for counting Ks (Kp). For the samples with an initial $\mathrm{OD}_{600}$ of $10^{-6}, 1$ $\mathrm{mL}$ of the mixed suspensions were directly plated without dilution on the GM17/ $\mathrm{Cm}$ plate supplemented with X-Gluc. For the samples with an initial $\mathrm{OD}_{600}$ of 10 ${ }^{-8}, 5 \mathrm{~mL}$ of the mixed suspensions were plated without dilution on the GM17/Cm plate supplemented with X-Gluc. During the period of incubation, the samples were taken to measure $\mathrm{OD}_{600}$ and the fraction of $\mathrm{Ks}(\mathrm{Kp})$ strain was calculated by counting the cells under microscope. For the ecosystem of $\mathrm{C} \alpha, \mathrm{C} \beta$ and $\mathrm{Kp}$, the above procedure remained the same except that the diluted co-culture samples were inoculated into $\mathrm{pH}$-defined media whose $\mathrm{pH}$ was adjusted every hour. Ten trials were performed for each initial condition.

Quantification of IcnA inhibition. To measure the inhibition from Ks to $\mathrm{Ca}$ or $\mathrm{C} \beta$, overnight Ks and $\mathrm{C} \alpha$ or $\mathrm{C} \beta$ monoculture were inoculated into fresh $\mathrm{GM} 17 / \mathrm{Cm} /$ Tween media at 1:1 ratio based on $\mathrm{OD}_{600}$. Then, the relative abundances of Ks were measured from the starting to $8 \mathrm{~h}$ of incubation with fluorescence microscope. The similar procedure was also used to determine the inhibition from $\mathrm{Kp}$ to $\mathrm{C} \alpha$ and $\mathrm{C} \beta$ in $\mathrm{pH}$-defined media ( $\mathrm{pH} \geq 7, \mathrm{pH} \leq 6$ and no $\mathrm{pH}$ control). Each culture condition has three replicates $(n=3)$

GusA3 enzyme assay. To directly visualize the Ks in three-strain system, GusA3 protein was used as a reporter to produce a blue green color. At the end of growth in three-strain consortium, a final concentration of $2 \mathrm{mM}$ of 5-bromo-4-chloro-3indolyl- $\beta$-D-glucuronide (X-Gluc) was added into the cultures and the blue green color formation was monitored. After incubation at $37^{\circ} \mathrm{C}$ for $1 \mathrm{~h}$, the supernatants were obtained by centrifuging the cultures at $10,000 \times \mathrm{g}$ for $10 \mathrm{~min}$ and images were taken

Mathematical modeling. In concert with the experimental ecosystem assembly, a bottom-up strategy was utilized to construct the dynamic models of the synthetic ecosystems. Briefly, we first created and characterized growth models of monocultures $(\mathrm{C} \alpha, \mathrm{C} \beta, \mathrm{Ks}, \mathrm{Kr}, \mathrm{Kp}$ ), then models for the cooperative $\mathrm{C} \alpha-\mathrm{C} \beta$ species as well as their interactions, and finally assembled individual modules into integrated models that represent complete ecosystems (e.g., $\mathrm{C} \alpha-\mathrm{C} \beta-\mathrm{Ks}, \mathrm{C} \alpha-\mathrm{C} \beta-\mathrm{Kr}$ and $\mathrm{C} \alpha-\mathrm{C} \beta$ $\mathrm{Kp}$ consortia). During model construction, ordinary differential equations were used to quantitatively describe the kinetics of three major classes of variables: nutrient availability, cell populations and bacteriocins mediating cellular interactions. Parameters were determined using data in the literature or by fitting the models to our experiments. MATLAB software was used to simulate the models, produce plots, and fit data for the models. A detailed description of the models is available in Supplementary Information.

Statistical analysis. All of the experiments were performed for multiple times. Replicate numbers of the experiments $(n)$ are indicated in the figure legends. Sample sizes were chosen based on standard experimental requirement in molecular biology. Data are presented as mean \pm s.d.

Reporting summary. Further information on research design is available in the Nature Research Reporting Summary linked to this article.

\section{Data availability}

Strains and plasmids constructed in this study are available from the corresponding author upon request. Data supporting the results in this paper are available within the paper and its supplementary information files. The source data of Figs. $2 \mathrm{~b}, \mathrm{c}, \mathrm{e}, 3 \mathrm{c}, \mathrm{d}, \mathrm{g}, \mathrm{h}$, 4a-h, 5b-d, 6d-6i, 7 and Supplementary Figs. 1a, 2c, 3, 5, 7b and 8-19 are provided as a Source Data file. All other relevant data are available from the author upon reasonable request.

\section{Code availability}

Custom MATLAB codes developed in this study are available from the corresponding author upon request. 
Received: 22 March 2019; Accepted: 9 December 2019;

Published online: 16 January 2020

\section{References}

1. Falkowski, P. G., Fenchel, T. \& Delong, E. F. The microbial engines that drive Earth's biogeochemical cycles. Science 320, 1034-1039 (2008).

2. Berendsen, R. L., Pieterse, C. M. \& Bakker, P. A. The rhizosphere microbiome and plant health. Trends Plant Sci. 17, 478-486 (2012).

3. Cho, I. \& Blaser, M. J. The human microbiome: at the interface of health and disease. Nat. Rev. Genet. 13, 260-270 (2012).

4. Widder, S. et al. Challenges in microbial ecology: building predictive understanding of community function and dynamics. ISME J. 10, 2557-2568 (2016).

5. Brenner, K., You, L. \& Arnold, F. H. Engineering microbial consortia: a new frontier in synthetic biology. Trends Biotechnol. 26, 483-489 (2008).

6. Johns, N. I., Blazejewski, T., Gomes, A. L. \& Wang, H. H. Principles for designing synthetic microbial communities. Curr. Opin. Microbiol. 31, 146-153 (2016).

7. Großkopf, T. \& Soyer, O. S. Synthetic microbial communities. Curr. Opin. Microbiol. 18, 72-77 (2014).

8. Mimee, M., Citorik, R. J. \& Lu, T. K. Microbiome therapeutics - advances and challenges. Adv. Drug Deliv. Rev. 105, 44-54 (2016).

9. Song, H. S., Cannon, W. R., Beliaev, A. S. \& Konopka, A. Mathematical modeling of microbial community dynamics: A methodological review. Processes 2, 711-752 (2014).

10. Cordero, O. X. \& Datta, M. S. Microbial interactions and community assembly at microscales. Curr. Opin. Microbiol. 31, 227-234 (2016).

11. Gonze, D., Coyte, K. Z., Lahti, L. \& Faust, K. Microbial communities as dynamical systems. Curr. Opin. Microbiol. 44, 41-49 (2018).

12. Zomorrodi, A. R. \& Segrè, D. Synthetic ecology of microbes: mathematical models and applications. J. Mol. Biol. 428, 837-861 (2016).

13. Faust, K. \& Raes, J. Microbial interactions: from networks to models. Nat. Rev. Microbiol. 10, 538-550 (2012).

14. Mounier, J. et al. Microbial interactions within a cheese microbial community. Appl. Environ. Microbiol. 74, 172-181 (2008).

15. Marino, S., Baxter, N. T., Huffnagle, G. B., Petrosino, J. F. \& Schloss, P. D. Mathematical modeling of primary succession of murine intestinal microbiota. Proc. Natl. Acad. Sci. USA 111, 439-444 (2014).

16. Stein, R. R. et al. Ecological modeling from time-series inference: insight into dynamics and stability of intestinal microbiota. PLoS Comput. Biol. 9, e1003388 (2013).

17. Garcia, F. C., Bestion, E., Warfield, R. \& Yvon-Durocher, G. Changes in temperature alter the relationship between biodiversity and ecosystem functioning. Proc. Natl Acad. Sci. USA 115, 10989-10994 (2018).

18. Ratzke, C. \& Gore, J. Modifying and reacting to the environmental $\mathrm{pH}$ can drive bacterial interactions. PLoS Biol. 16, e2004248 (2018).

19. Rivett, D. W. et al. Resource-dependent attenuation of species interactions during bacterial succession. ISME J. 10, 2259-2268 (2016).

20. Hart, S. F. M. et al. Uncovering and resolving challenges of quantitative modeling in a simplified community of interacting cells. PLoS Biol. 17, e3000135 (2019).

21. LaSarre, B., McCully, A. L., Lennon, J. T. \& McKinlay, J. B. Microbial mutualism dynamics governed by dose-dependent toxicity of cross-fed nutrients. ISME J. 11, 337-348 (2017).

22. Bachmann, H., Molenaar, D., Kleerebezem, M. \& van Hylckama Vlieg, J. E. High local substrate availability stabilizes a cooperative trait. ISME J. 5, 929-932 (2011).

23. Liu, J., Wu, C., Huang, I. H., Merritt, J. \& Qi, F. Differential response of Streptococcus mutans towards friend and foe in mixed-species cultures. Microbiology 157, 2433-2444 (2011).

24. Nguyen, A. T., Jones, J. W., Ruge, M. A., Kane, M. A. \& Oglesby-Sherrouse, A. G. Iron depletion enhances production of antimicrobials by Pseudomonas aeruginosa. J. Bacteriol. 197, 2265-2275 (2015).

25. Kreth, J., Merritt, J., Shi, W. \& Qi, F. Competition and coexistence between Streptococcus mutans and Streptococcus sanguinis in the dental biofilm. J. Bacteriol. 187, 7193-7203 (2005).

26. Liu, F., Mao, J., Lu, T. \& Hua, Q. Synthetic, context-dependent microbial consortium of predator and prey. ACS Synth. Biol. 8, 1713-1722 (2019).

27. Andrade-Domínguez, A., Salazar, E., Vargas-Lagunas, M., Kolter, R. \& Encarnación, S. Eco-evolutionary feedbacks drive species interactions. ISME J. 8, 1041-1054 (2014).

28. Buffie, C. G. et al. Precision microbiome reconstitution restores bile acid mediated resistance to Clostridium difficile. Nature 517, 205-208 (2015).

29. Palmer, A. C., Angelino, E. \& Kishony, R. Chemical decay of an antibiotic inverts selection for resistance. Nat. Chem. Biol. 6, 105-107 (2010).
30. van der Putten, W. H., Klironomos, J. N. \& Wardle, D. A. Microbial ecology of biological invasions. ISME J. 1, 28-37 (2007).

31. Kuipers, O. P., Beerthuyzen, M. M., de Ruyter, P. G., Luesink, E. J. \& de Vos, W. M. Autoregulation of nisin biosynthesis in Lactococcus lactis by signal transduction. J. Biol. Chem. 270, 27299-27304 (1995).

32. Coburn, P. S., Pillar, C. M., Jett, B. D., Haas, W. \& Gilmore, M. S. Enterococcus faecalis senses target cells and in response expresses cytolysin. Science 306, 2270-2272 (2004).

33. Kaern, M., Elston, T. C., Blake, W. J. \& Collins, J. J. Stochasticity in gene expression: from theories to phenotypes. Nat. Rev. Genet. 6, 451-464 (2005).

34. Elowitz, M. B., Levine, A. J., Siggia, E. D. \& Swain, P. S. Stochastic gene expression in a single cell. Science 297, 1183-1186 (2002).

35. Kiviet, D. J. et al. Stochasticity of metabolism and growth at the single-cell level. Nature 514, 376-379 (2014).

36. Ramkrishna, D. \& Mahoney, A. W. Population balance modeling. Promise for the future. Chem. Eng. Sci. 57, 595-606 (2002).

37. Shu, C. C., Chatterjee, A., Dunny, G., Hu, W. S. \& Ramkrishna, D. Bistability versus bimodal distributions in gene regulatory processes from population balance. PLoS Comput. Biol. 7, e1002140 (2011).

38. Hellweger, F. L. \& Bucci, V. A bunch of tiny individuals-Individual-based modeling for microbes. Ecol. Model 220, 8-22 (2009).

39. Klitgord, N. \& Segre, D. Environments that induce synthetic microbial ecosystems. PLoS Comput. Biol. 6, el001002 (2010).

40. Resat, H., Bailey, V., McCue, L. A. \& Konopka, A. Modeling microbial dynamics in heterogeneous environments: Growth on soil carbon sources. Microb. Ecol. 63, 883-897 (2012).

41. Shou, W., Ram, S. \& Vilar, J. M. Synthetic cooperation in engineered yeast populations. Proc. Natl. Acad. Sci. USA 104, 1877-1882 (2007).

42. Gore, J., Youk, H. \& van Oudenaarden, A. Snowdrift game dynamics and facultative cheating in yeast. Nature 459, 253-256 (2009).

43. Balagaddé, F. K. et al. A synthetic Escherichia coli predator-prey ecosystem. Mol. Syst. Biol. 4, 187 (2008).

44. Ozgen, V. C., Kong, W., Blanchard, A. E., Liu, F. \& Lu, T. Spatial interference scale as a determinant of microbial range expansion. Sci. Adv. 4, eaau0695 (2018).

45. Mee, M. T., Collins, J. J., Church, G. M. \& Wang, H. H. Syntrophic exchange in synthetic microbial communities. Proc. Natl. Acad. Sci. USA 111, E2149-E2156 (2014).

46. Kerr, B., Riley, M. A., Feldman, M. W. \& Bohannan, B. J. Local dispersal promotes biodiversity in a real-life game of rock-paper-scissors. Nature $\mathbf{4 1 8}$, 171-174 (2002).

47. Chuang, J. S., Rivoire, O. \& Leibler, S. Simpson's paradox in a synthetic microbial system. Science 323, 272-275 (2009).

48. Wintermute, E. H. \& Silver, P. A. Emergent cooperation in microbial metabolism. Mol. Syst. Biol. 6, 407 (2010).

49. Youk, H. \& Lim, W. A. Secreting and sensing the same molecule allows cells to achieve versatile social behaviors. Science 343, 1242782 (2014).

50. West, S. A., Griffin, A. S., Gardner, A. \& Diggle, S. P. Social evolution theory for microorganisms. Nat. Rev. Microbiol. 4, 597-607 (2006).

51. Wingreen, N. S. \& Levin, S. A. Cooperation among microorganisms. PLoS Biol. 4, 1486-1488 (2006).

52. Celiker, H. \& Gore, J. Cellular cooperation: insights from microbes. Trends Cell Biol. 23, 9-15 (2013).

53. Mirkovic, N. et al. Lactococcus lactis LMG2081 produces two bacteriocins, a non lantibiotic and a novel lantibiotic. Appl. Environ. Micobiol 82, 2555-2562 (2016).

54. Moll, G. et al. Lactococcin $\mathrm{G}$ is a potassium ion-conducting, two-component bacteriocin. J. Bacteriol. 178, 600-605 (1996).

55. Kong, W., Kapuganti, V. S. \& Lu, T. A gene network engineering platform for lactic acid bacteria. Nucleic Acids Res. 44, e37 (2016).

56. Bentley, W. E., Mirjalili, N., Andersen, D. C., Davis, R. H. \& Kompala, D. S. Plasmid-encoded protein: The principal factor in the "metabolic burden" associated with recombinant bacteria. Biotechnol. Bioeng. 35, 668-681 (1990).

57. Glick, B. R. Metabolic load and heterologous gene expression. Biotechnol. Adv. 13, 247-261 (1995).

58. Lidstrom, M. E. \& Konopka, M. C. The role of physiological heterogeneity in microbial population behavior. Nat. Chem. Biol. 6, 705-712 (2010).

59. Stegen, J. C., Lin, X., Konopka, A. E. \& Fredrickson, J. K. Stochastic and deterministic assembly processes in subsurface microbial communities. ISME J. 6, 1653-1664 (2012).

60. Masel, J. Genetic drift. Curr. Biol. 21, R837-R838 (2011).

61. Hallatschek, O., Hersen, P., Ramanathan, S. \& Nelson, D. R. Genetic drift at expanding frontiers promotes gene segregation. Proc. Natl Acad. Sci. USA 104, 19926-19930 (2007)

62. Fernández, A., Horn, N., Gasson, M. J., Dodd, H. M. \& Rodríguez, J. M. Highlevel coproduction of the bacteriocins nisin A and lactococcin A by Lactococcus lactis. J. Dairy Res. 71, 216-221 (2004). 
63. Callanan, M. J., Russell, W. M. \& Klaenhammer, T. R. Modification of Lactobacillus $\beta$-glucuronidase activity by random mutagenesis. Gene $\mathbf{3 8 9}$, 122-127 (2007).

64. Madsen, S. M., Arnau, J., Vrang, A., Givskov, M. \& Israelsen, H. Molecular characterization of the $\mathrm{pH}$-inducible and growth phase-dependent promoter P170 of Lactococcus lactis. Mol. Microbiol. 32, 75-87 (1999).

65. Cody, M. L. \& Diamond, J. M. Ecology and evolution of communities. Nature 260, 204 (1976).

66. Gotelli, N. J. How do communities come together? Science 286, 1684-1685 (1999).

67. Weiher, E. \& Keddy, P. Ecological assembly rules: perspectives, advances, retreats. Cambridge University Press (2001).

68. Le Loir, Y., Gruss, A., Ehrlich, S. D. \& Langella, P. A nine-residue synthetic propeptide enhances secretion efficiency of heterologous proteins in Lactococcus lactis. J. Bacteriol. 180, 1895-1903 (1998).

69. Zhu, D. et al. Isolation of strong constitutive promoters from Lactococcus lactis subsp. lactis N8. FEMS Microbiol. Lett. 362, fnv107 (2015).

70. Egbert, R. G. \& Klavins, E. Fine-tuning gene networks using simple sequence repeats. Proc. Natl. Acad. Sci. USA 109, 16817-16822 (2012).

\section{Acknowledgements}

We thank Prof. Jon Nissen-Meyer and Prof. Todd Klaenhammer for their generous gifts of DNA materials. This work was supported by the National Science Foundation (1553649), Office of Naval Research (N000141612525) and Department of Energy (DE-SC0019185). F.L. was supported by the China Scholarship Council. J.M. was supported by the China Scholarship Council and the National Natural Science Foundation of China (11575059 and 11847315). Q.H. was supported by the Research Program of the State Key Laboratory of Bioreactor Engineering and 111 Project (B18022).

\section{Author contributions}

T.L. conceived the project; T.L., Q.H., Y.F. and R.B. designed research; F.L. performed experiments; J.M. developed mathematical models; W.K. contributed new reagents/ analytic tools; F.L., J.M. and T.L. analyzed data; F.L., J.M. and T.L. wrote the paper with input from all other authors.

\section{Competing interests}

The authors declare no competing interests.

\section{Additional information}

Supplementary information is available for this paper at https://doi.org/10.1038/s41467019-13986-6.

Correspondence and requests for materials should be addressed to T.L.

Peer review information Nature Communications thanks the anonymous reviewers for their contribution to the peer review of this work.

Reprints and permission information is available at http://www.nature.com/reprints

Publisher's note Springer Nature remains neutral with regard to jurisdictional claims in published maps and institutional affiliations.

(c) (i)

Open Access This article is licensed under a Creative Commons Attribution 4.0 International License, which permits use, sharing, adaptation, distribution and reproduction in any medium or format, as long as you give appropriate credit to the original author(s) and the source, provide a link to the Creative Commons license, and indicate if changes were made. The images or other third party material in this article are included in the article's Creative Commons license, unless indicated otherwise in a credit line to the material. If material is not included in the article's Creative Commons license and your intended use is not permitted by statutory regulation or exceeds the permitted use, you will need to obtain permission directly from the copyright holder. To view a copy of this license, visit http://creativecommons.org/ licenses/by/4.0/.

(C) The Author(s) 2020 